Final Report

for

\author{
DOE Contract DE-AC02-76-ET53017
}

\title{
On the Physics of Ion Ring Generation for the Stabilization of FRX Discharges
}

\author{
by \\ Hans Fleischmann \\ School of Applied and Engineering Physics, Cornell University, 205 Clark Hall \\ Ithaca, NY 14853
}

\begin{abstract}
As envisioned in the respective proposals, our work over the final periods of this contract centered on experimental and related theoretical investigations of the physics of the generation of ion rings which might be used for a tilt stabilization of FRX rings, with experiments centering around the new MICE equipment constructed during the earlier parts of the work. In particular, our work mainly consisted of the following: (i) investigations concerning the operation of the ion beam driver constructed earlier, especially of the magnetically insulated ion diode and possible improvements of that diode, (ii) theoretical analyses on the propagation and neutralization of the ion beam during its injection into the confinement tank, and (iii) experimental investigations on the physics of ion beam injection and ring formation in the MICE device; in these latter experiments very significant discrepancies of various observed data and those predicted from single-particle orbit calculations were found. Unfortunately, the work could not be completed due to stop in funding, but a semiquantitative analysis of these discrepancies indicated insufficient space charge charge neutralization of the ion beam in the tank and/or charge-exchange effects in the diode are considered the most likely reasons.In contrast to claims by its authors, a similar discrepancy also was found in the published data of the earlier IREX experiment. The results of our investigations, which - together with the design and construction of the equipment - provided the basis for two $\mathrm{PhD}$ theses, will be briefly described in this report; the full details can be obtained from the two theses.




\section{DISCLAIMER}

This report was prepared as an account of work sponsored by an agency of the United States Government. Neither the United States Government nor any agency thereof, nor any of their employees, make any warranty, express or implied, or assumes any legal liability or responsibility for the accuracy, completeness, or usefulness of any information, apparatus, product, or process disclosed, or represents that its use would not infringe privately owned rights. Reference herein to any specific commercial product, process, or service by trade name, trademark, manufacturer, or otherwise does not necessarily constitute or imply its endorsement, recommendation, or favoring by the United States Government or any agency thereof. The views and opinions of authors expressed herein do not necessarily state or reflect those of the United States Government or any agency thereof. 


\section{DISCLAIMER}

Portions of this document may be illegible in electronic image products. Images are produced from the best available original document. 


\section{INTRODUCTORY SUMMARY}

As envisioned in the respective proposals, our work over the final periods of this contract centered on experimental and related theoretical investigations of the physics of the generation of ion rings which might be used for a tilt stabilization of FRX rings, with experiments centering around the new MICE equipment constructed during the earlier parts of the work. In particular, our work mainly consisted of the following: (i) investigations concerning the operation of the ion beam driver constructed earlier, especially of the magnetically insulated ion diode and possible improvements of that diode, (ii) theoretical analyses on the propagation and neutralization of the ion beam during its injection into the confinement tank, and (iii) experimental investigations on the physics of ion beam injection and ring formation in the MICE device; in these latter experiments very significant discrepancies of various observed data and those predicted from single-particle orbit calculations were found. Unfortunately, the work could not be completed due to stop in funding, but a semiquantitative analysis of these discrepancies indicated insufficient space charge charge neutralization of the ion beam in the tank and/or charge-exchange effects in the diode are considered the most likely reasons.In contrast to claims by its authors, a similar discrepancy also was found in the published data of the earlier IREX experiment. The results of our investigations, which - together with the design and construction of the equipment - provided the basis for two $\mathrm{PhD}$ theses ${ }^{1}$, will be briefly described in this report; the full details can be obtained from the two theses.

\section{ION GENERATION}

As mentioned above, the investigations of the report period were performed using our new water/oil-insulated positive-voltage 1-MV-2-Ohm-200-nsec pulsed-power driver and a ring-shaped

\footnotetext{
1 Note: S. E. Jones, "High-Power Accelerator and Magnetically Insulated lon Diode for Ion Ring Studies", PhD Thesis, Cornell University, and W. J. Podulka, "On the Formation of Large-Orbit Proton Rings by Cusp Injection", PhD Thesis, Cornell University.
} 
magnetically insulated passive-anode ion diode. Of these, the pulser had been obtained by an extensive minimum-budget $(\leq 50 \mathrm{k} \$)$ reconfiguration of our old oil-insulated, negative-voltage 5MV-60-Ohm-60-nsec RECE pulse driver. Also, the ion diode was reconfigured and improved during this period to incorporate suggestions resulting from various model calculations also performed by us during that period. The design and operational test and behavior of these components will be described in the following.

\section{(A) Pulse Driver}

Our new negative-voltage 1-MV-2-Ohm-200-nsec pulse driver was obtained by reconfiguring the Marx generator as well as the fast-pulsed section. The final design of the two sections is indicated in Figs. 1 and 2.

In the still-oil-insulated Marx generator, essentially the old capacitor arrangement with 56 80-kV capacitors and the old trigger circuitry was used; however, the old single-chain circuitry was re-polarized, split into two sections that were connected in parallel. Both chains were triggered by feeding the old trigger unit into both chains. In order to avoid the usual parallelization problems, the new inductive insulation transformer shown in Fig. 3 was used which induced a triggerenhancing additional voltage pulse in the slower Marx chain whenever the other chain completed its erection. Using this arrangement, the typical errection delays between the two chains were reduced to easily-sufficient $150 \mathrm{nsec}$.

The new water-insulated fast-pulse section consists of two 2-Ohm-200-nsec pulseline sections, the first of which first is charged from the Marx generator through the equally new oilwater interface over a (1/4)-cycle time of about $1 \mu \mathrm{sec}$. On reaching the voltage peak, the $\mathrm{SF}_{6}$ insulated switch connects the charged pulse-forming line through the second pulse transfer line to the ion diode.

The expected pulse shapes for this arrangement were calculated using various computer codes for a variety of diode arrangements, and a good agreement with experimentally observed pulse shapes was obtained as typified in Fig. 4 


\section{(B) Ion Diode}

Work on the ion diode during the final periods concentrated on possible improvements over the magnetically-insulated diode configuration used in our earlier experiments. The final design of the "new" diode configuration was obtained through extensive particle-in-cell code calculations of the electron and ion flow in various diode configurations; subsequently this diode was tested experimentally.

\section{(1) "Old" Diode Configuration}

In our earlier experiments, the "passive-field-shaper" diode arrangement shown in Fig. 5 was used. In this case, the insulating magnetic field was generated by a single coil embedded into the ring-shaped anode. In the vicinity of the actual diode gap the magnetic field was compressed and enhanced by two passive copper rings positioned on the cathode side in front of the supporting aluminum plate. A virtual cathode was formed in front of the ion generating anode surface by electron emission from two thin, concentric stainless-steel rings outside and inside the beam channel. The magnetic field distribution in the diode gap could be influence mainly by the relative width of the copper rings, the extension of the stainless-steel rings and the axial position of the anode plate. As anode, passive flashboard surfaces of various designs were used.

Experimental measurements using the new driver showed not-quite-sufficient diode currents of less than $200 \mathrm{kA}$, typical proton fluences of $\leq 15 \mu \mathrm{Asec} / \mathrm{cm}^{2}$, and diode efficiencies $\leq 30 \%$. In addition, the usefulness of the diode for beam injection measurements was further limited by rather large shot-to-shot fluctuations (typically factor 2) and similar azimuthal asymmetries and fluctuations. Therefore, this diode configuration was abandoned in favor of a diode configuration with additional field coils positioned on the cathode side. 


\section{(2) New-Configuration Diode}

(a) Basic Configuration: The basic design of the new diode configuration is shown in Fig. 6 (Final design). As mentioned, the insulating magnetic field now is generated by a set of three concentric coils - one embedded in the anode and two on the cathode side (one each inside and outside the beam channel). The three coils are energized independently in order to permit larger changes of the field shape in the gap. The passive copper rings used in the "old" diode were retained, but the remaining cathode surface now was moved away from the aluminum plate; a foil changer was used to separate vacuum-wise the diode region from the downstream injection tank. Various magnetic field configurations achievable with a three-coil configuration are illustrated in Fig. 7a. ( $\alpha$ denotes the ratio of outer-cathode-coil current to anode-coil current. In this case, a ratio, $\rho \approx 1$, of the inner to outer cathode-coil currents was used.) As indicated, a separatrix now separates the inner B-field region surrounding the anode coil from two outer regions, namely (i) one where the field lines remain in the cathode region, and (ii) one where the field lines connect the cathode to the anode stock. Of these, (i) is particularly important since it will tend to retain and concentrate electrons coming into the diode gap from the cathode parts outside the diode; the related negative space charge obviously will help to enhance the ion flow in the diode. (In the "old" diode design where all field lines surrounded the anode, any electrons reaching the gap generally will be lost much more rapidly along the field lines to the anode stock.) In addition, as visible in the ( $\alpha=$ 4)-section of Fig. 6, enhanced cathode-coil currents now will permit some field lines originating on the outer parts of the cathode plate to reach the anode surface; thus, electrons generated on these field lines will tend to bombard directly the flashover surface.

(b) Design Using PIC-Code Calculations: The final design of the new diode was obtained by extensive particle-in-cell model calculations using the MAGIC code for a wide variety of spatial arrangements, coil currents, and voltages. Examples of code results are shown in Fig. $7 \mathrm{~b}$ 
which shows the expected enhancements of the electron clouds in the diode gap and some of the electrons travelling to the anode stock along the separatrix, as well as electrons proceeding directly to the flashover surface. For the choice of the final spatial diode design, various criteria, in particular the variability of the magnetic configuration with coil current, obtainable magnetic field strengths, obtained ion current densities, anode bombardment etc. were used; code limitations suppressed angular asymmetries as well as most instabilities, thus limiting the reality of the model; however, the code clearly showed the main features of interest. Very interestingly, the code results - after the initial build-up period - showed a steadily rising ion current seemingly related to the continuing inflow of electrons into the gap permitting an increasing ion density in the gap. A field configuration for the final design configuration is shown in Fig. 8. The new diode arrangement together with the related capacitor banks etc. was constructed, and incorporated into the device. Electric test measurements of coil currents, magnetic fields, etc. agreed with the calculated values.

(c) Diode Operation: Subsequently, the operation of the diode was tested quite extensively. Examples of the recorded diode voltages, currents, ion beam currents, and Faraday ion current signals are shown in Fig. 9. Overall, the obtained gross operation of the diode was sizably improved: Ion current densities increased by about a factor 4 to over $350 \mathrm{~A} / \mathrm{cm}^{2}$, with an efficiency of about $50 \%$ and fluences reaching $60 \mu \mathrm{Asec} / \mathrm{cm}^{2}$. Also, shot-to-shot fluctuations decreased to about $20 \%$, and uniformity of the ion current around the diode ring improved very markedly and became reproducible. Finally, the physics of the diode, in particular the dependency of the diode parameters on operating parameters agreed quite well with those expected from our code calculations.

\section{ION BEAM INIIECTION}

Investigations of the physics of ion ring generation through the injection strong ion beams mainly proceeded along two paths: (i) theoretical calculations of ion orbits in various magnetic 
cusp-field configurations relevant to the MICE tank - and their optimization - were performed; in addition, also semiquantitative analyses of various other potential problems of ion beam injection were made as well as preliminary orbit calculations on magnetic field models as they will be encountered during FRX injection. (ii) Experimental measurements with related theoretical analyses were performed in which the ring-shaped ion beam generated by our ion beam diode was injected into the magnetic field of the MICE tank.

\section{(A) Basic MICE Configuration}

An view of the overall MICE device is given in Fig. 10, showing the pulse driver ("Marx generator" and "pulse former") on the right ending on its left in the ion diode from which the ringshaped ion beam then is injected into the MICE tank on the far left. The magnetic field in the MICE tank basically consists of a pulsed homogeneous field that is separated by a thick aluminum plate from the diode region and its magnetic field. An example of the resulting total magnetic field configuration in the diode-injection region is shown in Fig. 11. Overall, the magnetic flux and field of the diode coils provides one side of magnetic cusp field the other side of which is made up by the magnetic field of the MICE tank. As is well known, ions launched axially from the diode surface - while propagating through the cusp-field region - will be turned in $\theta$-direction so that they will encircle the axis. At proper strengths of both parts of the cusp field, single ion orbits may be fully turned and even reflected in the tank field. It is this latter characteristic that may lead to the formation of self-confined ion rings whenever sufficiently many ions will encircle the axis and thus generate a strong ring current with a corresponding self-magnetic-field. Consequently, the optimization goal consists in finding magnetic field configurations for which a maximum self-field will be produced in the MICE tank, i.e. for which a maximum fraction of the ions generated in the diode (with the actual operating parameters and their time dependencies) will simultaneously contribute to the ring current. This problem also can be complicated by the electric fields generated 
by the only-partially-neutralized injected beam; here, due to the very large ion beam densities, even very small non-neutralizations can very seriously influence the ion orbits and, thus, the beam behavior. Correspondingly, both the potential charge neutralization and the particle orbits in the combined fields were considered extensively, as is described in the following.

\section{(B) Beam Charge Neutralization}

Basically, space charge neutralization of the ion beam is required within the diode channel (and its vicinity) as well as in the MICE tank itself.

Within the diode channel, the perpendicular (to the beam) direction of the magnetic field and the nearness of the channel walls permits electrons emitted from the walls (either by electric fields or ion bombardment) to quickly enter the beam. Corresponding estimates and model calculations indicate that sufficient space charge neutralization should be obtained to cancel up to $99 \%$ of the ion charge, reducing related electric potentials and fields to levels which will not seriously distort the beam beam propagation.

Unfortunately, similarly good neutralization in vacuum certainly cannot be expected in the regions away from the direct diode channel and even more so in the MICE tank where much fewer neutralizing electrons can be expected to be generated at the wall, and where these electrons will have to travel a much larger distance; also, the ion orbits in this section are more sensitive to electric fields. Here, it becomes important that the thin mylar foil spanned over the opening of the field-separating aluminum plate permits the tank to be filled with a background gas up to a few Torr without influencing the diode operation. In principle, space charge neutralization then will be enhanced through collisional ionization of the gas by the beam ions. However, since the basic ionization process is charge-neutral, beam neutralization still will require gas ions to leave the beam region - either along or across the B-field - and/or to generate new electrons nearer to the wall. Correspondingly, overall ionization densities much larger than the beam density will be required, 
necessitating the presence and consideration of cascade ionizations etc.. Only then will the electric fields be shifted to the beam surface in a sufficiently short time. Our estimates and model calculations indicate that gas densities of at least a few mTorr will be required for beam neutralization. Additional considerations of the use of a pre-generated background plasma in the MICE tank as an alternative indicate required plasma densities, $\mathrm{n} \geq 10^{13}-10^{14} \mathrm{~cm}^{-3}$.

\section{(B) Single-Particle Orbit Simulations:}

Extensive orbit calculations were performed for a variety of magnetic field configurations, in particular including some idealized fields, the earlier IREX experiment (of another group) and the field of the MICE device. For each configuration, particular emphasis was given to the "cut-off" energy (at which the particle was reflected back into the diode region) and the extreme orbit parameters (at which a particle might get lost to the wall or other obstacles), and their dependence on various orbit parameters, like radius-of-birth on the ion diode and the initial deviation of the orbit angle from the anode surface normal.

First assuming no electric field, we found that the cut-off energy will depend sizably on the radius-of-birth on the ion diode, and generally will result in a quite significant spread of axial velocities, $\mathrm{v}_{\mathrm{par}}$, in the homogeneous parts of the field. Fig. 12 shows an example for the IREX field with $d v_{\mathrm{par}} / \mathrm{v}_{\mathrm{par}} \approx 50 \%$ ! In this light, the self-confinement of the "rings" claimed by the experimenters appears highly surprising, to say the least. Furthermore, the cut-off energies also depend quite sizably on the angle, $\alpha$, between the initial ion velocity and the anode surface normal. Fig. 13 gives an example for a typical MICE field. For that configuration, optimal operating parameters then were obtained by considering the extreme orbit parameters, spread in cut-off angle etc. as a function of the diode parameters. Fig.14 gives an example.

In some additional calculations, also the influence of potential electric fields - in the diode 
region as well as the tank region - was considered. As indicated by the results shown in Fig. 15, radial electric fields of up to $50 \mathrm{kV} / \mathrm{cm}$ in the diode region certainly appear managable. In contrast, radial electric field of a few $\mathrm{kV} / \mathrm{cm}$ in the tank region generally will drive the particles to the wall or will sizably change the cut-off energy.

\section{(C) Model Calculations of Single-Particle Injection into FRX Rings}

For injection of ions into an FRX ring, generally a combination of established ion rings with the FRX is anticipated. Unfortunately, however, earlier plasma code simulations already have indicated that such ion rings - when propagating in a background plasma tend to separate from their self-confining field so that a large percentage of the ions will perform single-particle orbits in the FRX field. Correspondingly, such single-particle orbits become very relevant. As shown in the example of Fig. 16, it unfortunately appears that only a rather small fraction of any single ions propagating towards an FRX ring become trapped in the FRX for more than a few bounces, even when considering plasma drag. As a result, the injection of ion rings into FRX rings may require $\mu$ sec-time scale changes of the FRX field to obtain good trapping, and thus may be sizably more difficult than generally assumed. (see Fig. 17 for example)

\section{MUCE INJECTUON EXPERIMENTS}

The goal of our MICE experiments was to perform detail tests some of the basic premises of the physics of ion beam injection and ring formation under real circumstances. Earlier simulations

of other groups using highly idealized assumptions regarding the magnetic vacuum field, $\mathrm{B}_{\mathrm{vac}}$, the ion beam (mono-energetic, mono-directional, full cylindrical uniformity, full space-charge neutralization etc.), and the plasma, had predicted trapping efficiencies of $\geq 90 \%$. High efficiencies seemingly were confirmed by some of the IREX and other experiments of that group which 
claimed the formation of rings with $\mathrm{dB} / \mathrm{B} \leq 4-10 \%$ in good agreement with particle orbit calculations. Besides these physics goals, the MICE experiments also aimed at obtaining ion rings with as-high-as-possible self-field ratios, $\delta \mathrm{B}_{\text {ring }} / \mathrm{B}_{\mathrm{vac}}$.

In view of the physics goals of the experiment, a rather wide range of diagnostic was incorporated into the MICE device. Using these diagnostics, injection experiments were performed and then compared with single-particle predictions. Unfortunately, the entire process had to be cut short due to the stop of funding; in the following, the experiments and the related analyses will be described briefly. In summary, the experiments performed and analyzed clearly indicates a very sizable (approximately a factor of 10 !) discrepancy between the experimentally observed $\delta \mathrm{B} / \mathrm{B}$ values and those predicted; various possible reasons for this discrepancy were analyzed with nonneutralization and beam clumping appearing the most likely. A maximum self-field value of $d B / B \approx$ $11 \%$ was obtained in the experiments.

A similar detail analysis also was applied to the published IREX data, and revealed a commensurate discrepancy of about a factor 5 , unfortunately in striking disagreement with the above quoted claims.

\section{(A) MICE-Device and_Diagnostic}

The MICE device already has been described above. The diagnostic used for the injection experiments included:

(a) The usual machine diagnostic for diode voltage, $V_{d}$, and diode current, $I_{d}$, both with an accuracy of about $5 \%$.

(b) Ion Diode Region: (Fig. 18)

(i) Faraday cups to measure the ion beam density;

(ii) Symmetric set of pick-up loops for measuring the total ion beam current;

(iii) Carbon-activation for measuring proton fraction; here particular attention was given to 
minimizing and determining the signal reduction due to target blow-off;

(c) Cusp Region after Diode: (Fig. 19)

Another set of diagnostics (i)-(iii) was installed in the cusp region.

(d) Downstream Region in Tank:

(i) A radial set of Faraday cups (directed about $60^{\circ}$ to the axis direction) was used to measure the density of axis-encircling ions;

(ii) A set of magnetic pick-up coils (4 along axis, 2 off-axis) were used to measure the size and spatial distribution, $\delta \mathrm{B}(\mathrm{x}, \mathrm{t})$, of the magnetic self-field generated by the ion beam/ring;

(iii) A shadow box with 4-5 radially spaced holes was used to measure the direction of the ions at the positions of the holes;

(iv) Carbon plates measured (with sizable statistical error) the time-integrated proton flux at various positions.

(B) Observations and Analysis:

\section{(a) Procedure}

A series of 12 shots with varying parameters was analyzed in detail. For this purpose, first the time-dependent orbitals in the externally applied magnetic field, $\mathrm{B}_{\mathrm{ex}}$, were calculated for ions generated in the diode at energies equal to the momentary voltage, $\mathrm{V}_{\mathrm{d}}(\mathrm{t})$, assuming full space charge neutralization (i.e. electric fields outside diode, $\mathrm{E}=0$ ), and no magnetic self-fields, $\delta \mathrm{B}=0$. Subsequently, the measured ion beam densities in the diode, the projected ion beam density distributions and the projected magnetic fields, $\mathrm{dB}$, generated by the beam downstream were calculated, and then compared with the observed distributions.

\section{(b) Results:}

(i) A number of discrepancies exist between the observed MICE data and the one predicted by orbital analysis. Fig. 20 shows the main problem, namely a much faster fall-off of the beam- 
generated field along the tank axis (already including an observed-but-not-fully explained decrease of the beam density between the diode region and the cusp region!), in particular from a distance of $45 \mathrm{~cm}$ into the tank. While the observed signals still are quite comparable with the predicted ones at $z=45 \mathrm{~cm}$, the ratio between the observed to the predicted signals is about 10 for $z \geq 70 \mathrm{~cm}$. This discrepancy also is confirmed by the smallness of the signals from the other beam diagnostics.

(ii) As mentioned an only slightly smaller discrepancy between the observed and predicted beamgenerated fields and ring behavior was found in the same analysis of the earlier IREX data.

\section{(c) Analysis of Potential Loss Reasons:}

Subsequently the potential reasons for the observed discrepancies were investigated by estimating the effects of various potential error sources. The result of these analyses can be summarized as follows:

(i) Deflection of the ions by E-fields in the diode: little effect up to projected fields $\mathrm{E} \approx 15-25$ $\mathrm{kV} / \mathrm{cm}$;

(ii) Scattering, recombination etc. in background gas: negligible;

(iii) Error in diode voltage: not significant for $\delta V_{d} / V_{d} \leq 3 \%$; $\delta V_{d} / V_{d} \leq-(15-20) \%$ could generate changes in $\delta \mathrm{B} / \mathrm{B}$ of the observed size; such error is certainly outside error bars of $V_{d}$, but might be explained by charge exchange losses inside diode which would also explain difficulties with activation measurements;

(iv) Plasma shielding of magnetic probes: factor 1.5 - 2 possible but no firm conclusions;

(v) Effect of beam-generated field, $\delta \mathrm{B}_{\text {self: }}$ negligible

(vi) Beam divergence in diode: most likely not significant; but can explain the fall-off in beam density between diode and cusp region;

(v) Axial E- fields (from non-neutralization of beam charge): $\mathrm{E} \approx 1-2 \mathrm{kV} / \mathrm{cm}$ in the cusp region would change orbits so as to explain the observed reduction in $\delta B_{\text {self }}$,

(vi) Radial E-fields: $E \approx$ few $\mathrm{kV} / \mathrm{cm}$ would could reduce $\delta B_{\text {self }}$ by factor $3 ; 4 \mathrm{kV} / \mathrm{cm}$ would keep beam from penetrating into homogeneous field region. 
(vii) Beam non-uniformity (in $\theta$-direction): no hard conclusion, probably not a large effect; Summary: The most likely main source(s) for the observed discrepancies are electric space charge fields from a non-complete neutralization of the beam charge (fields of the required magnitude could result from a charge imbalance of only $\approx 1 \%$ ), and/or from reduction of the average beam ion energy by charge transfer processes within the diode.

\section{(d) Suggested Future Work}

Based on the details of the above analysis, a number of suggestions were developed for future, which can be summarized as follows:

(i) E-Fields in Tank: A direct measurement of the electric fields existing in the tank during injection; such measurement will be difficult but would be highly worthwhile;

(ii) Actual Ion Energies: Increase Marx voltage to test changes in proton fraction and dB;

Measure with comparably lower $\mathrm{B}_{\text {tank }}$; additional direct ion energy measurements could be incorporated;

(iii) More detailed measurements: More complete determinations of radial current profiles, $\mathrm{L}(\mathrm{dI} / \mathrm{dt})$ corrections of voltage probe, etc. 


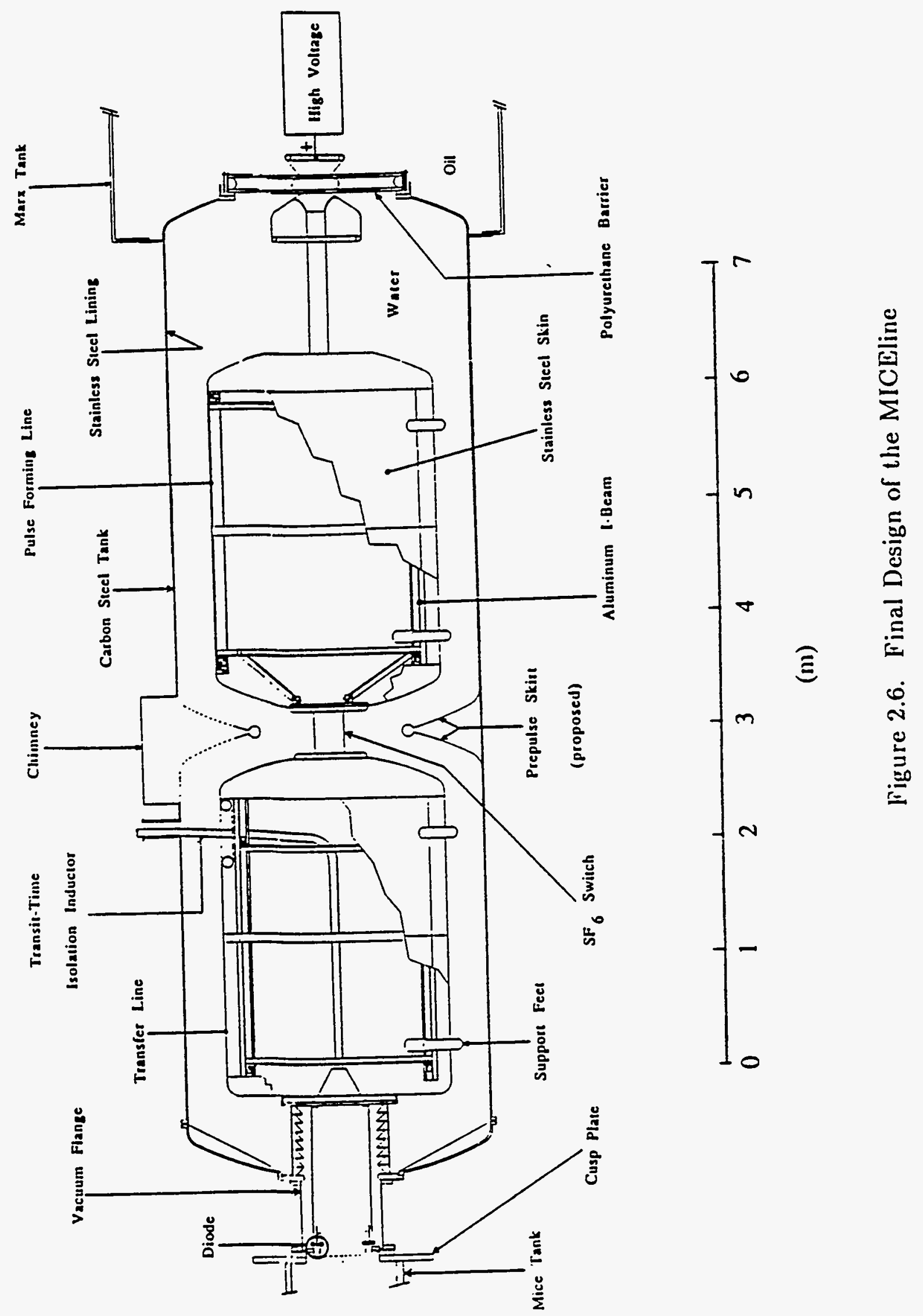

Fig 1 


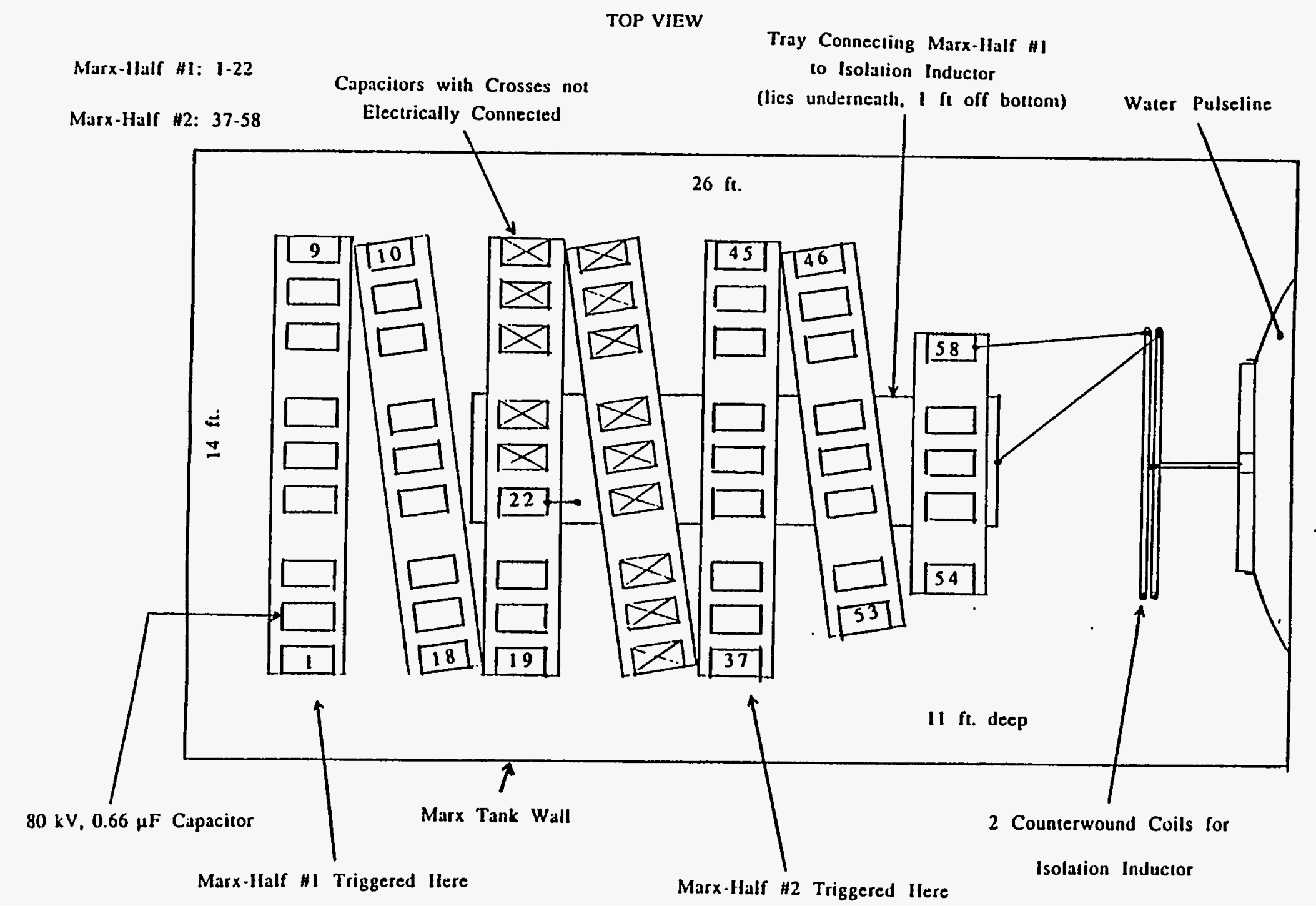

Figure 2.7. Marx Capacitor Arrangement. 


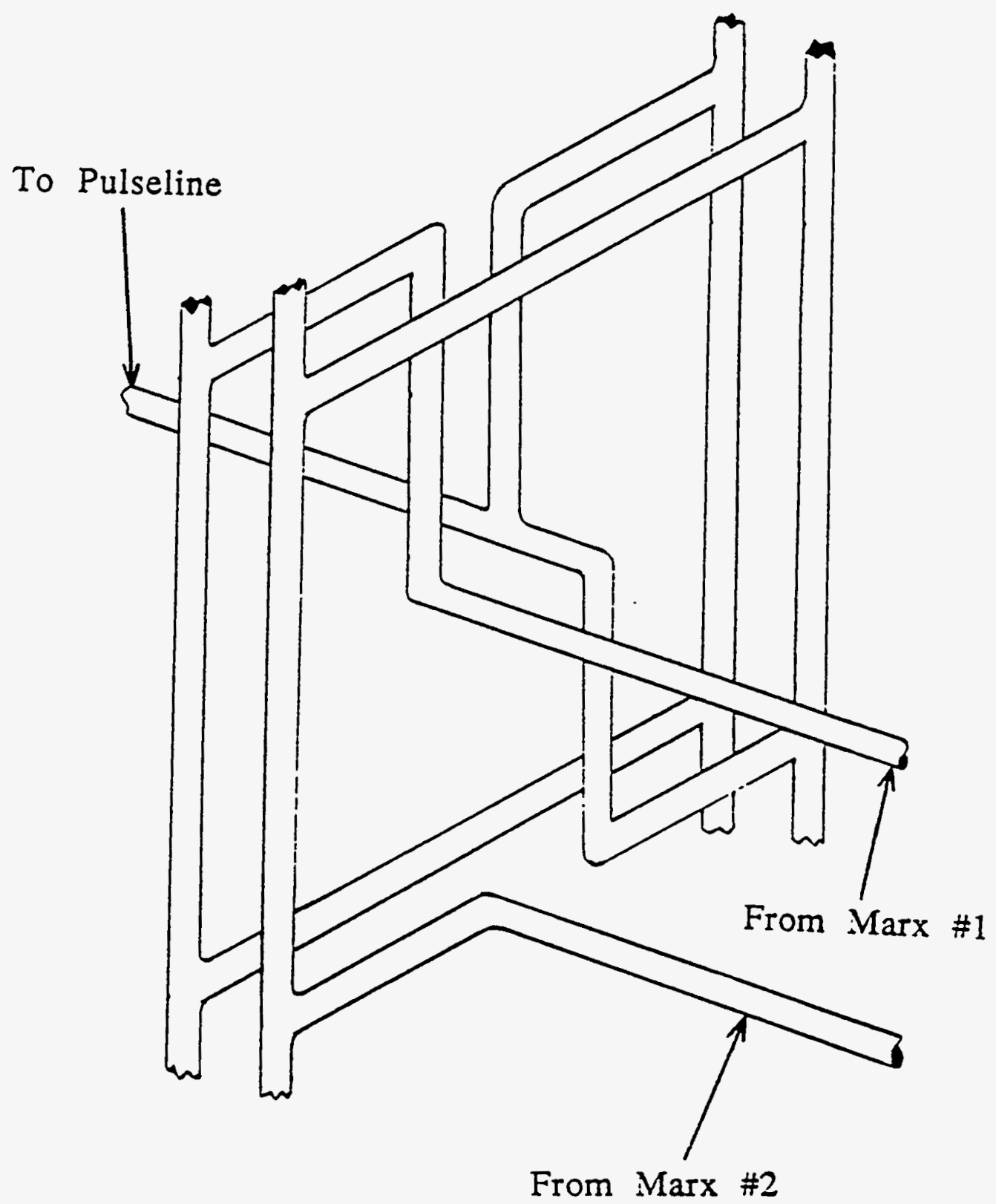

Figure 2.9. Diagram of Inductive Isolation Transformer 

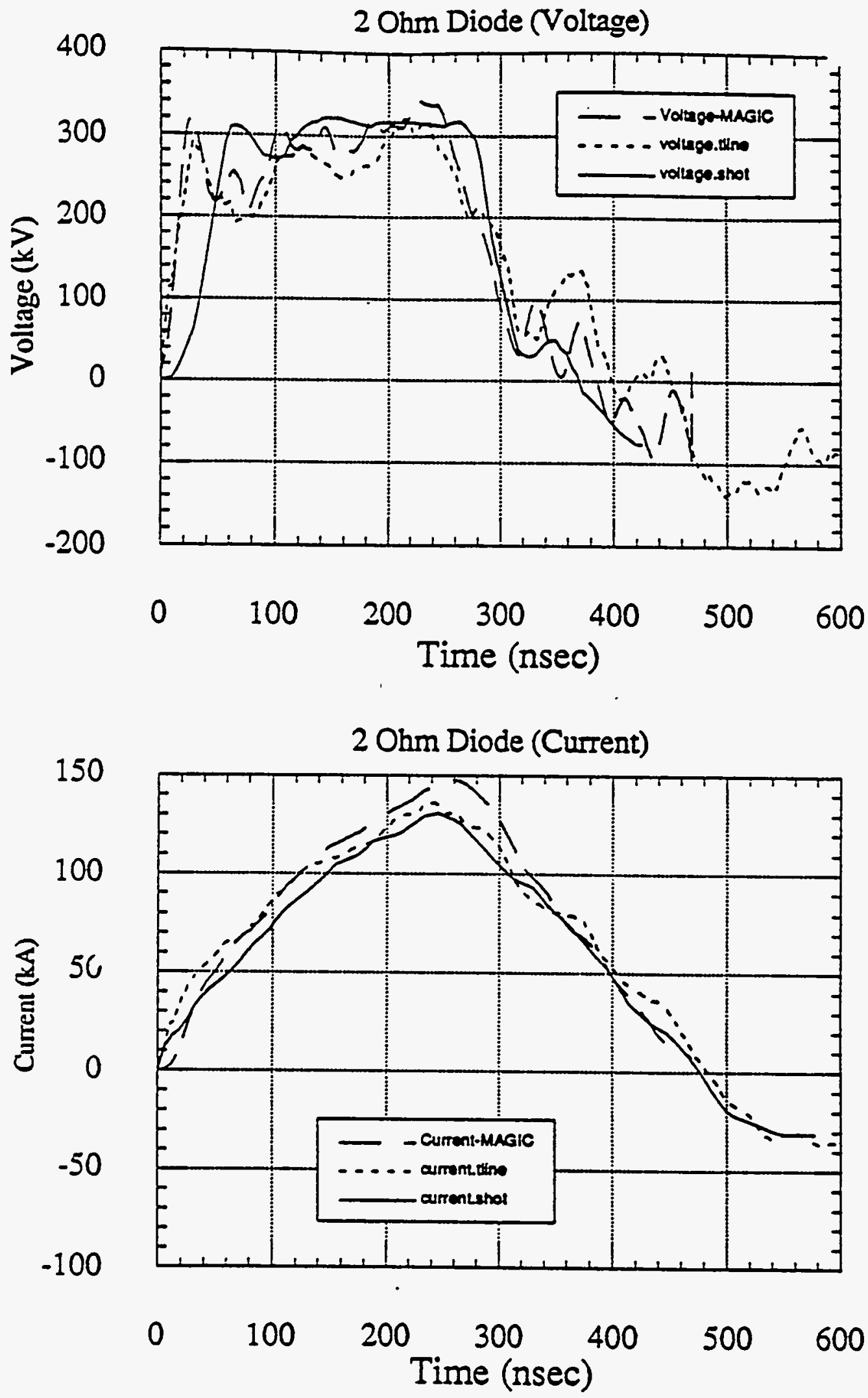

Figure 2.35. Accelerator test with a $2 \Omega$ diode. Top: diode voltage monitor. Bottom: diode current monitor. 


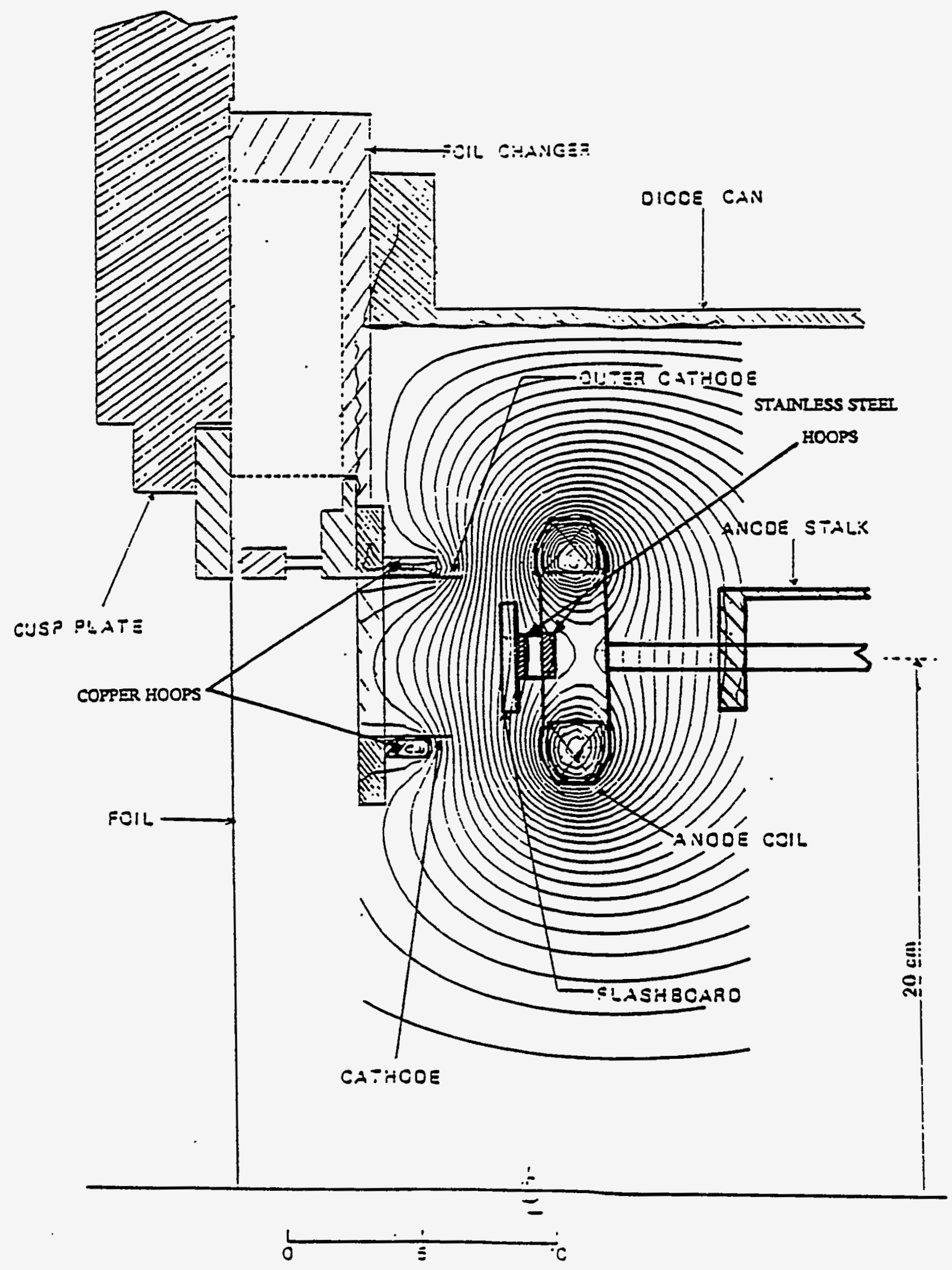

Figure 3.5. Physical and magnetic geometry of magnetically insulated ring diode without cathode coils 


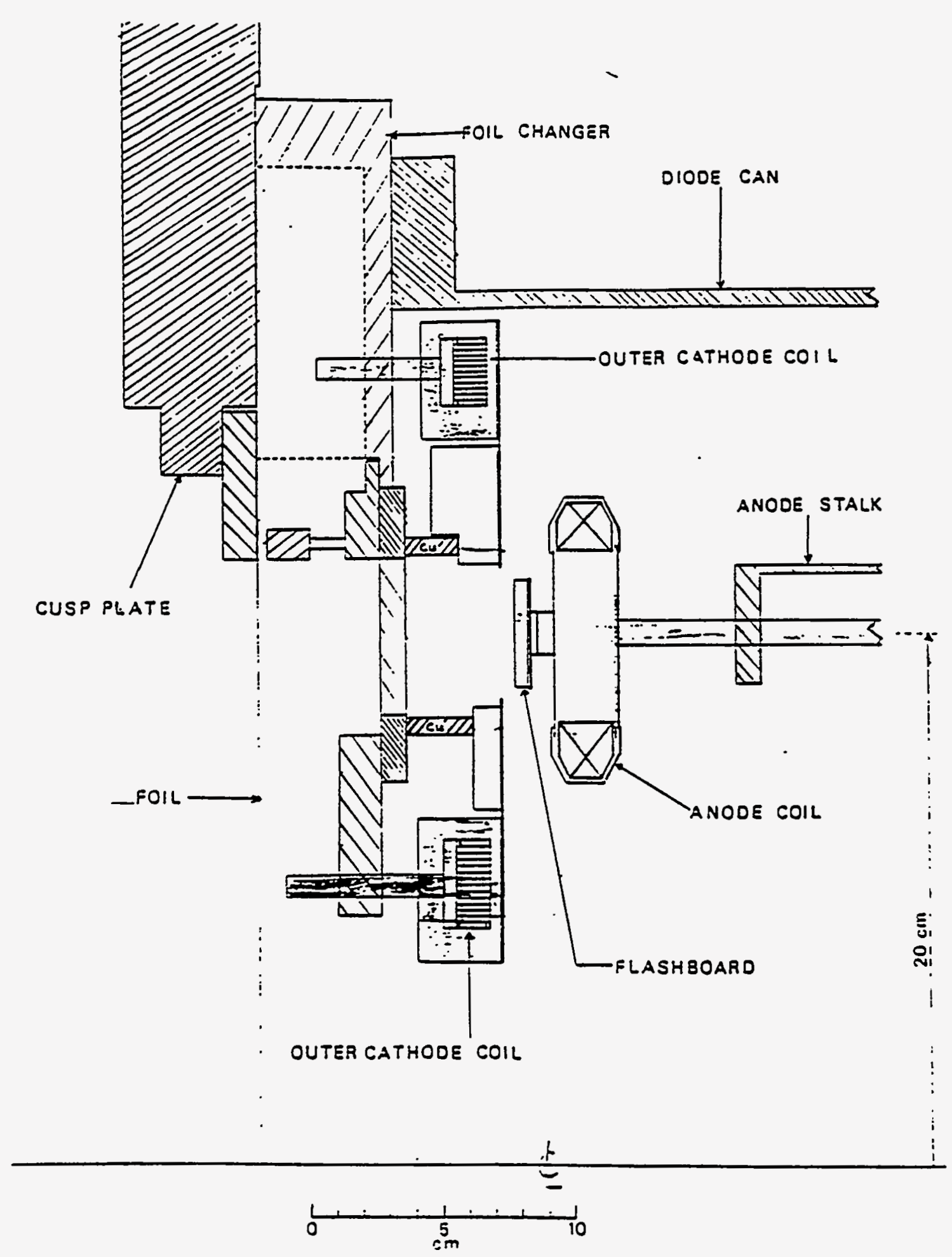

Figure 3.39. Physical geometry of new diode with anode-side and cathode-side coils. 

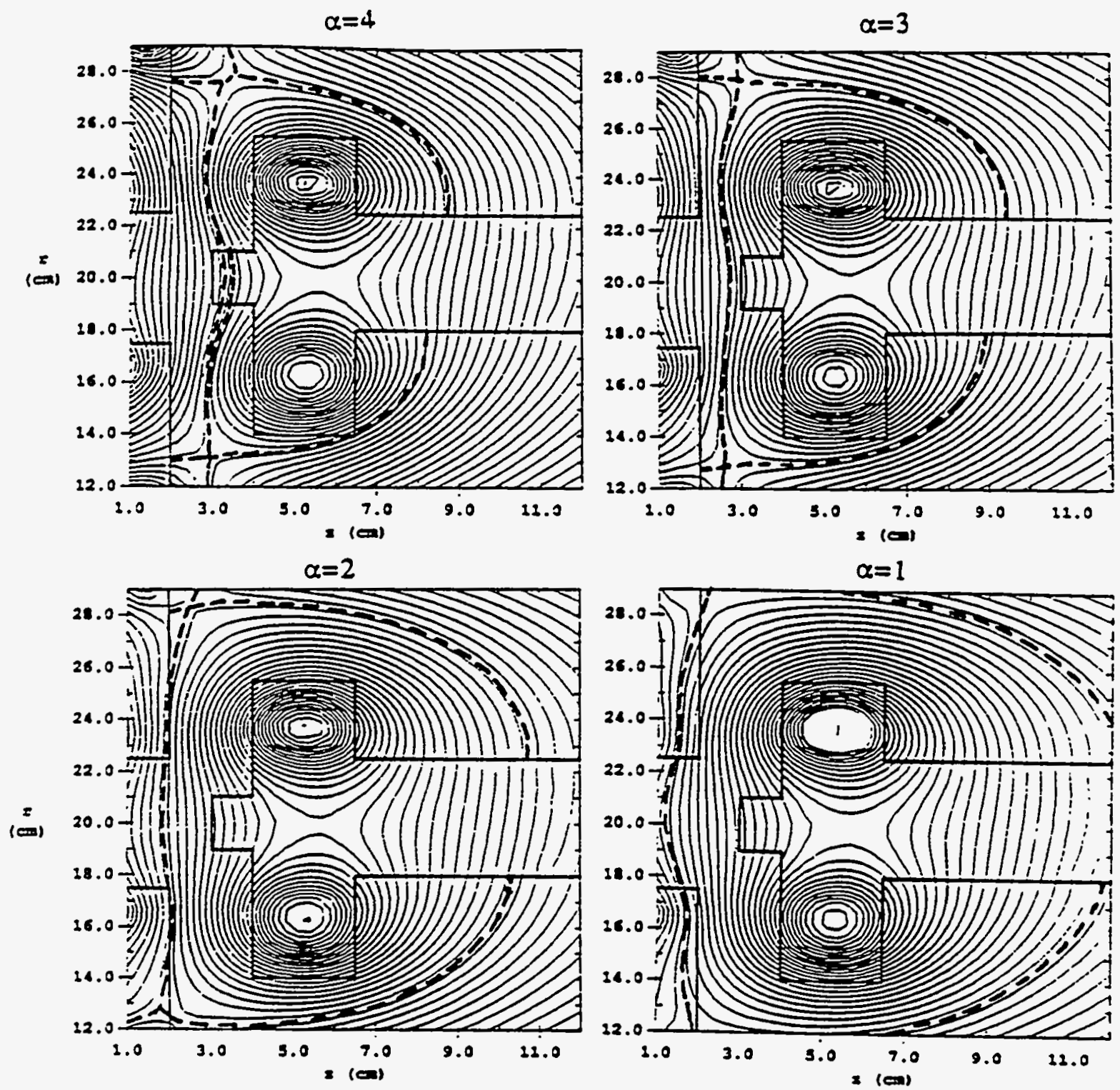

Figure 3.20. MSUPER plots of diode magnetic fields for $\alpha=4,3,2,1$. Dashed lines show pseudo-separatrices. 
A) $\alpha=4$

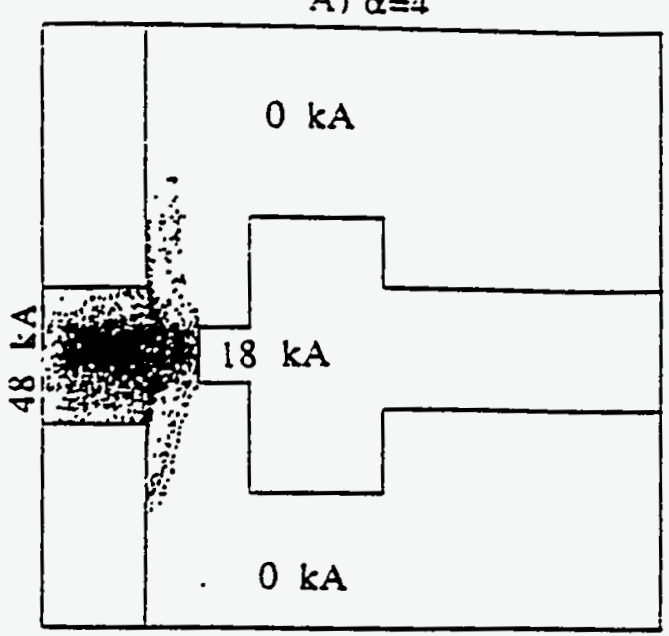

C) $\alpha=2$
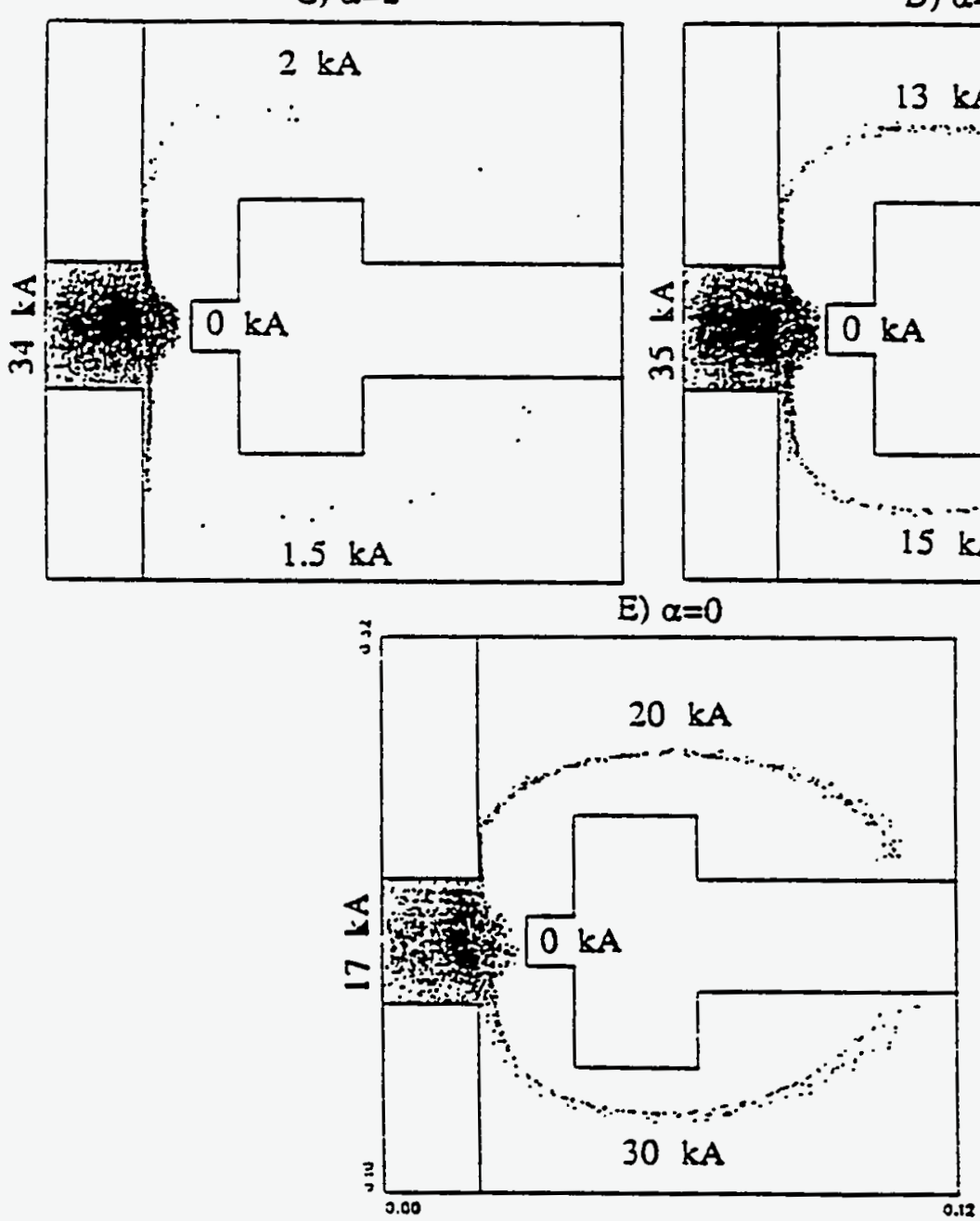

E) $\alpha=0$

B) $\alpha=3$

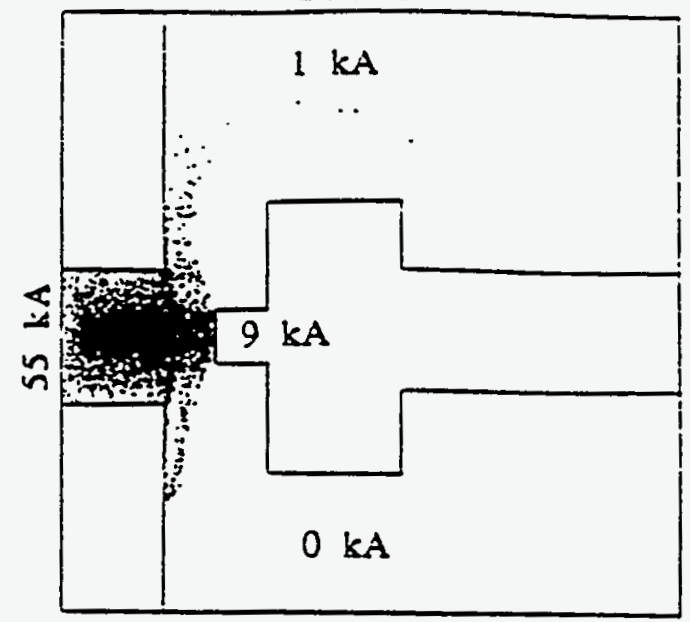

D) $\alpha=1$

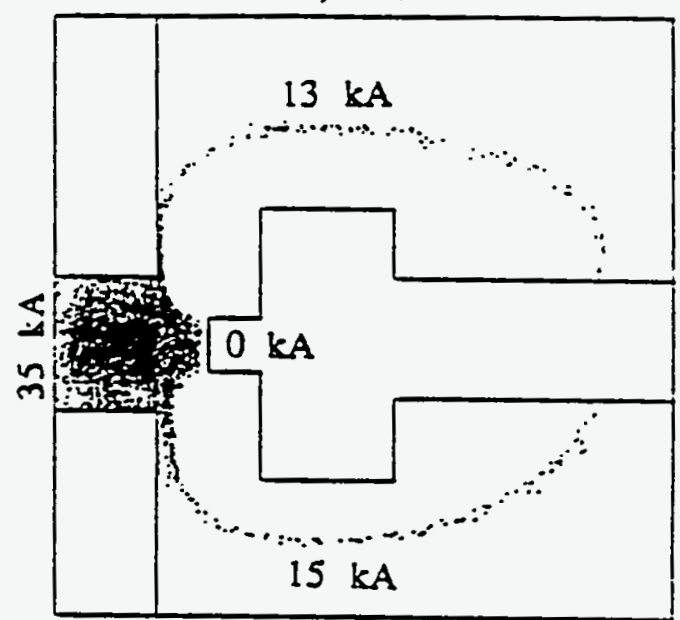




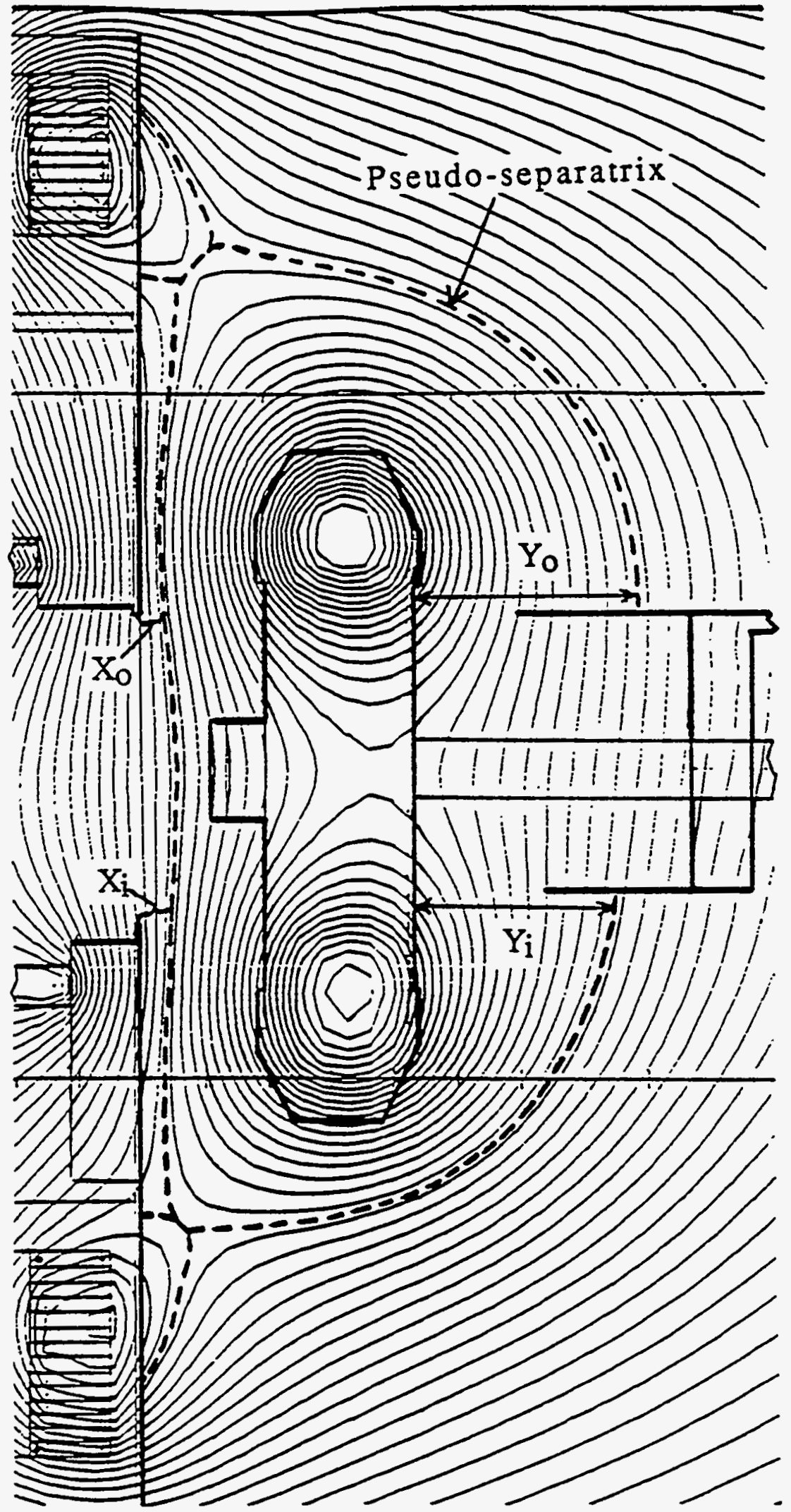

Figure 3.40. Example of magnetic geometry of new diode with $I_{\text {outer }} / I_{\text {inner }}=0.51$, and $\alpha=2.6$. 

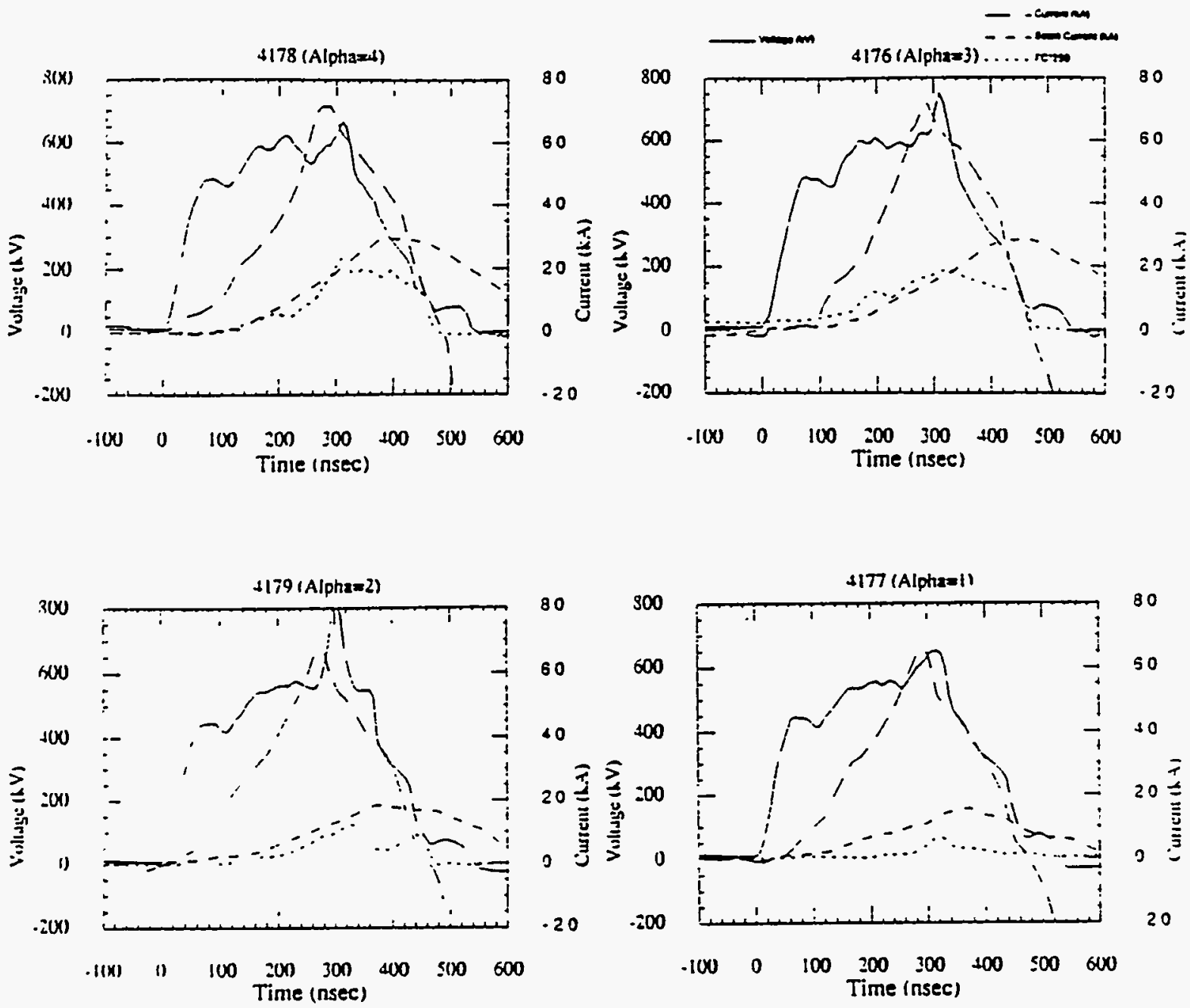

Figure 3.54. Effect of varying $\alpha$ on diode voltage and current (with epoxy in flashboard grooves). All shots at $B_{i n s}=4 \mathrm{kG}$. Fast-rising solid line is diode voltage, long dashed line is diode current, short dashed line is ion beam current, and slowrising solid line is Faraday cup current (multiplied by 250). 
$\overline{0.6 .4}$

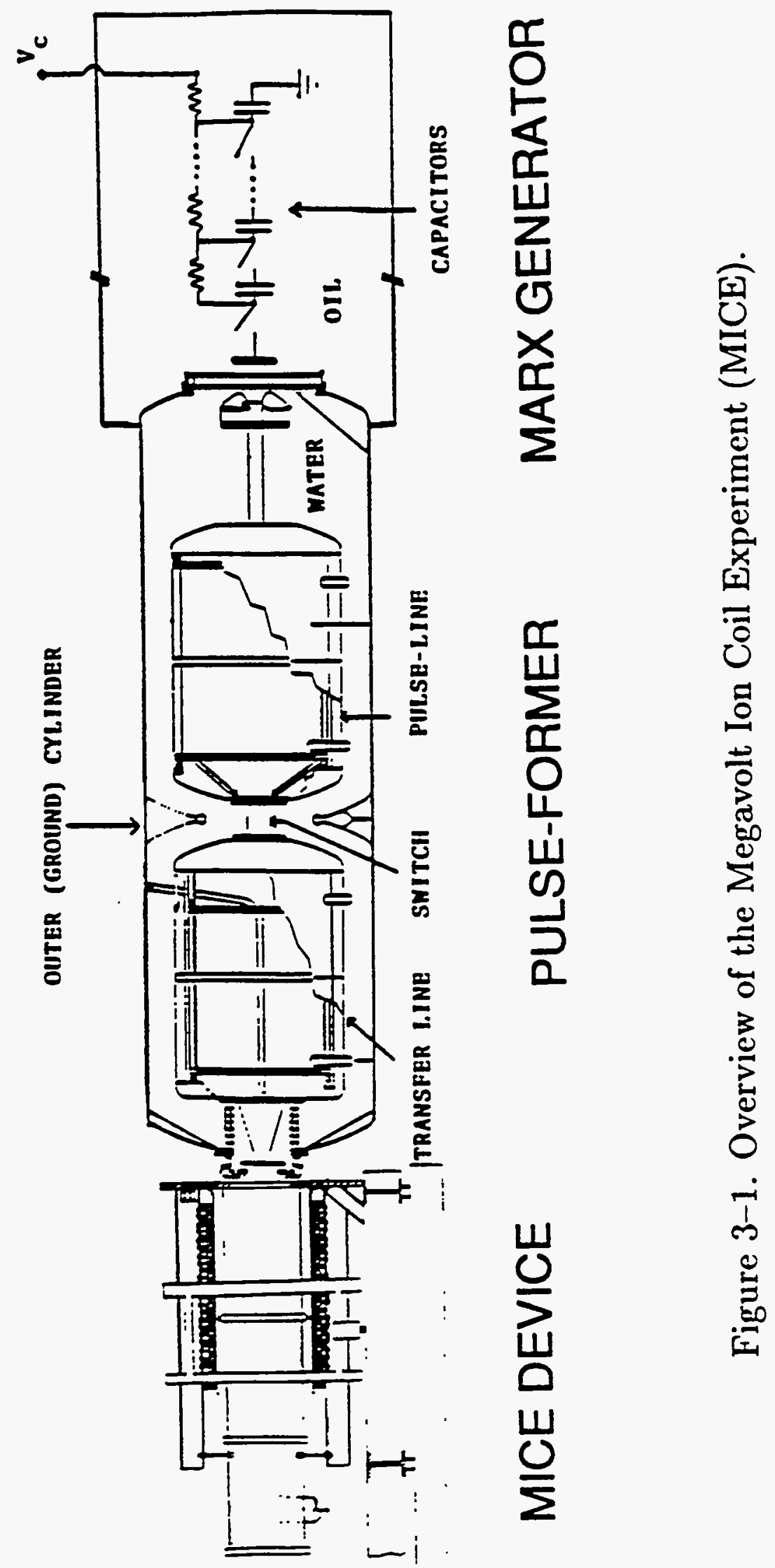




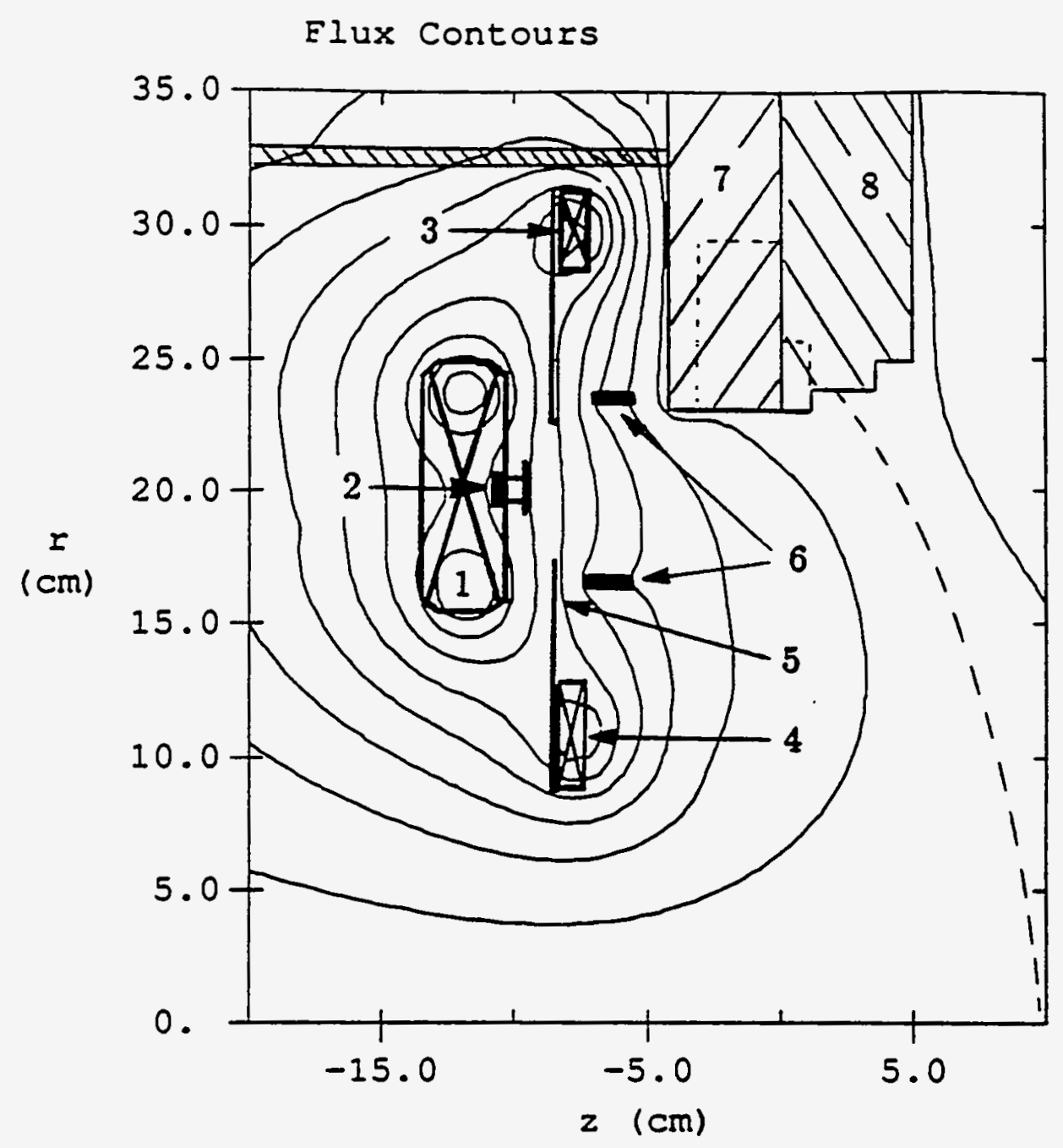

Figure 3-4. Flux plot showing field due to anode and cathode field coils in the diode region.

1. Anode Coil 5. Cathode

2. SS Field Shaping Hoop 6. Cu Field Shaping Hoop

3. Outer Cathode Coil 7. Foil Changer Plate

4. Inner Cathode Coil 8. Cusp Plate 

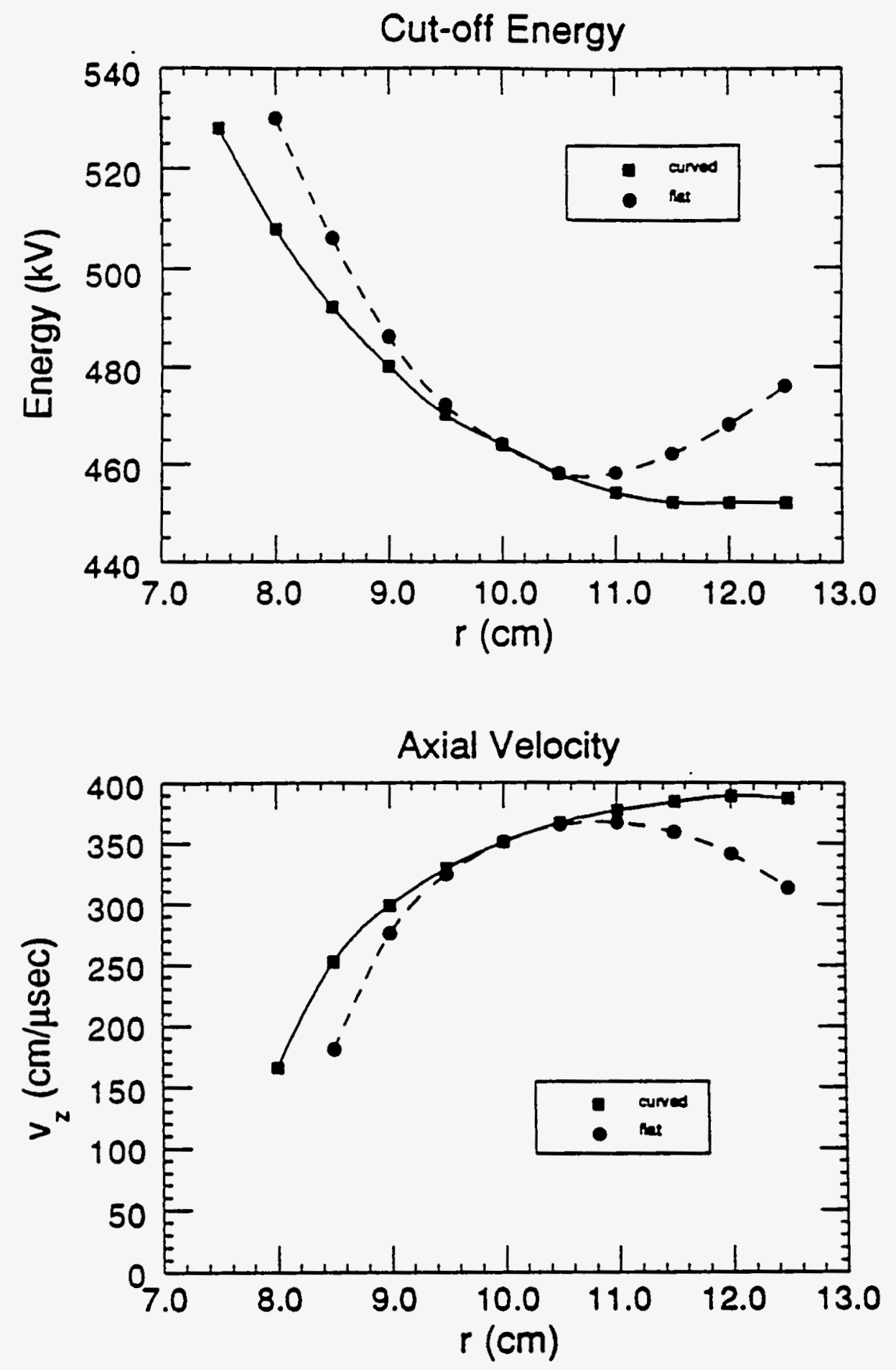

Figure 2-24. Cusp cut-off curve and resulting axial velocity spread for the IREX device. The "curved anode" is coincident with the flux surface passing through the middle of the anode.

Top: $\psi$ vs. $\mathrm{r}$ along the flat anode surface.

Middle: Cut-off energy as a function of radius.

Bottom: Axial velocity distribution for $v_{0}=1000 \mathrm{~cm} / \mu \mathrm{sec}$. 

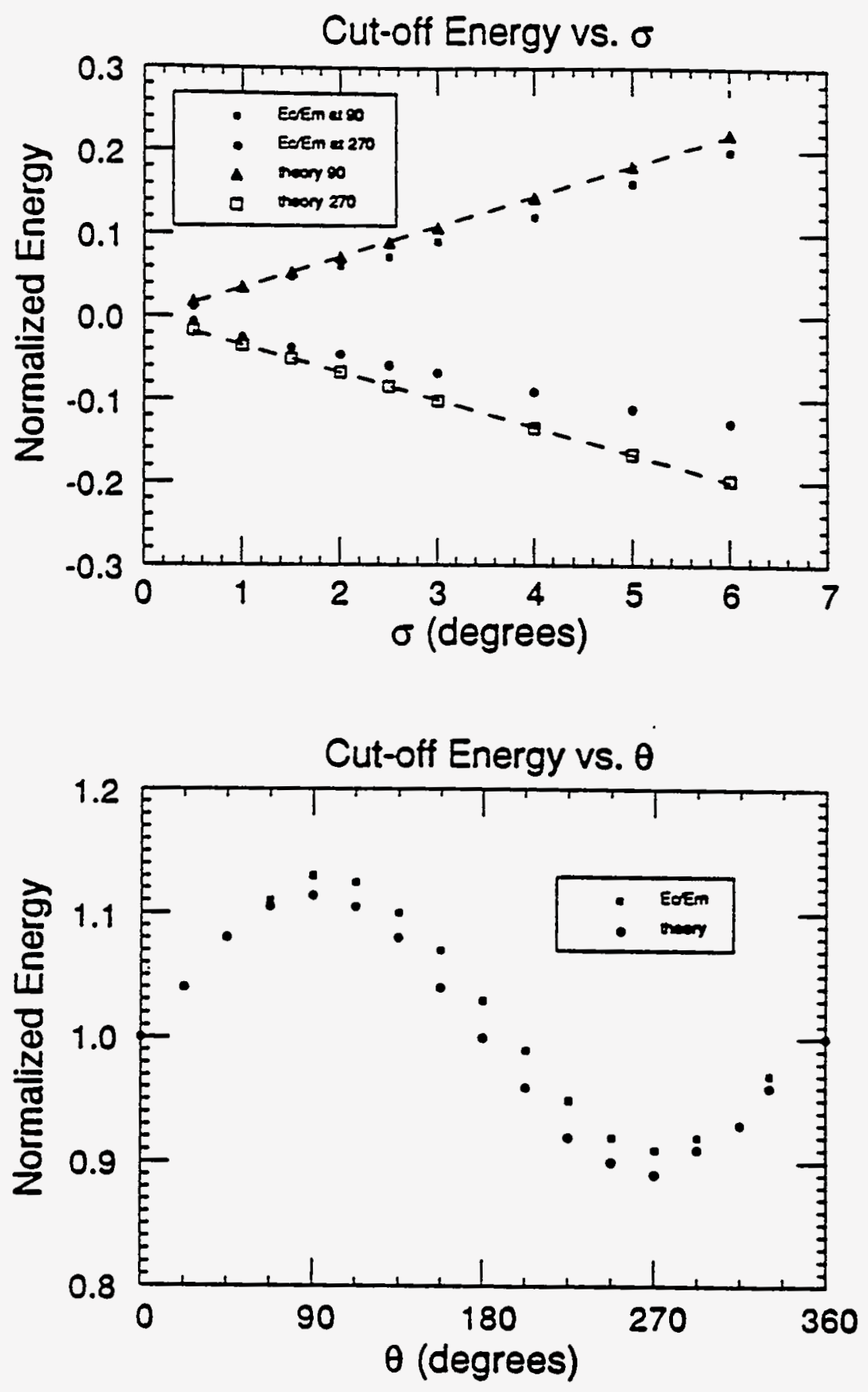

Figure 2-26. Dependence of cusp cut-off energy on beam divergence.

Top: Normalized cut-off energy vs. $\sigma$ for $\theta=90^{\circ}$ and $\theta=270^{\circ}$. Bottom: Normalized cusp cut-off energy vs. $\theta$ for $\sigma=4^{\circ}$ 


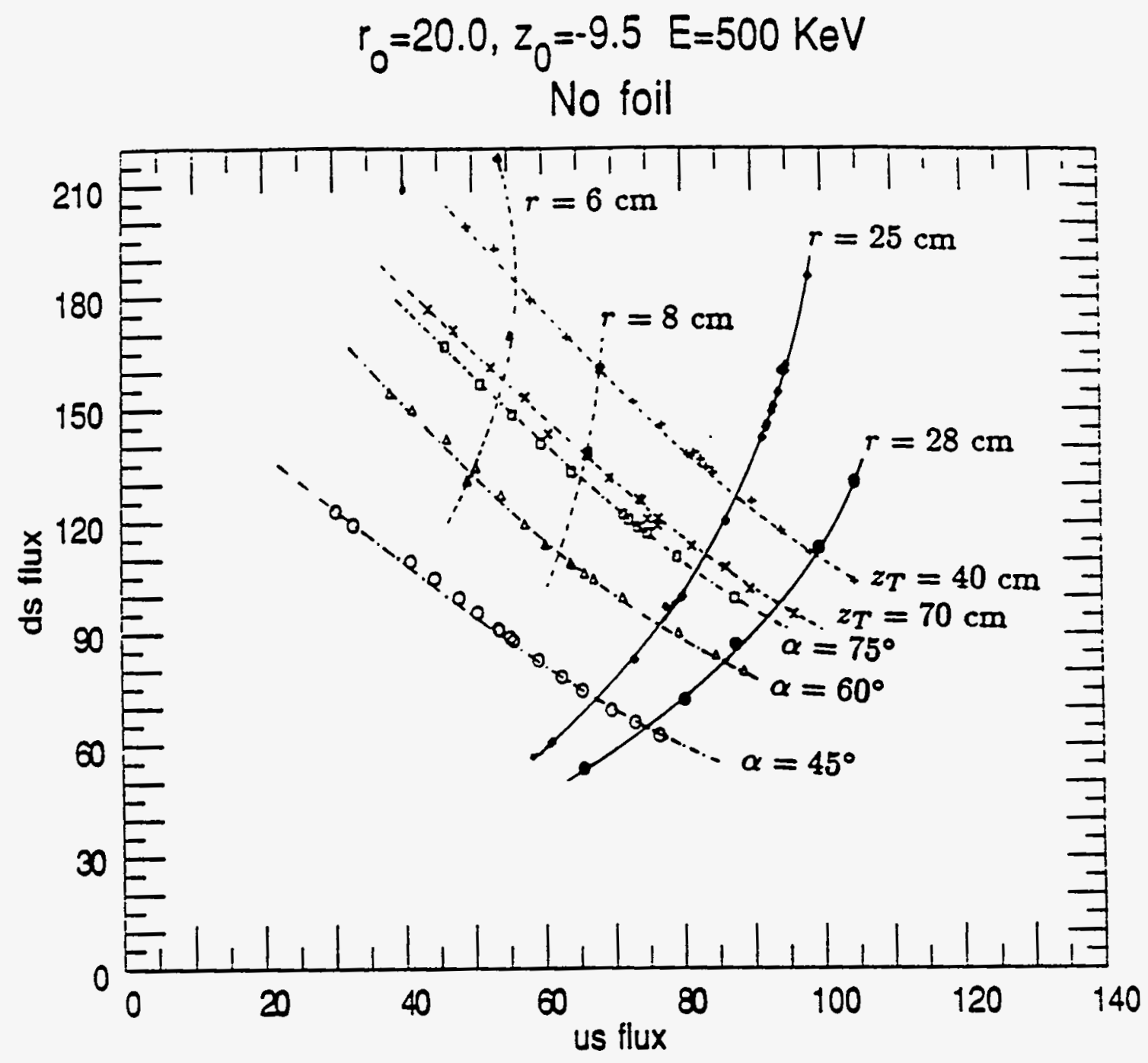

Figure 2-28. Operation space plot for a particle injected into the standard MICE configuration. Solid lines are curves of maximum radius, dashed lines are minimum radius, dotted lines are axial turning point, and dot-dash lines are particle pitch angle. The injection radius is $20 \mathrm{~cm}$. 

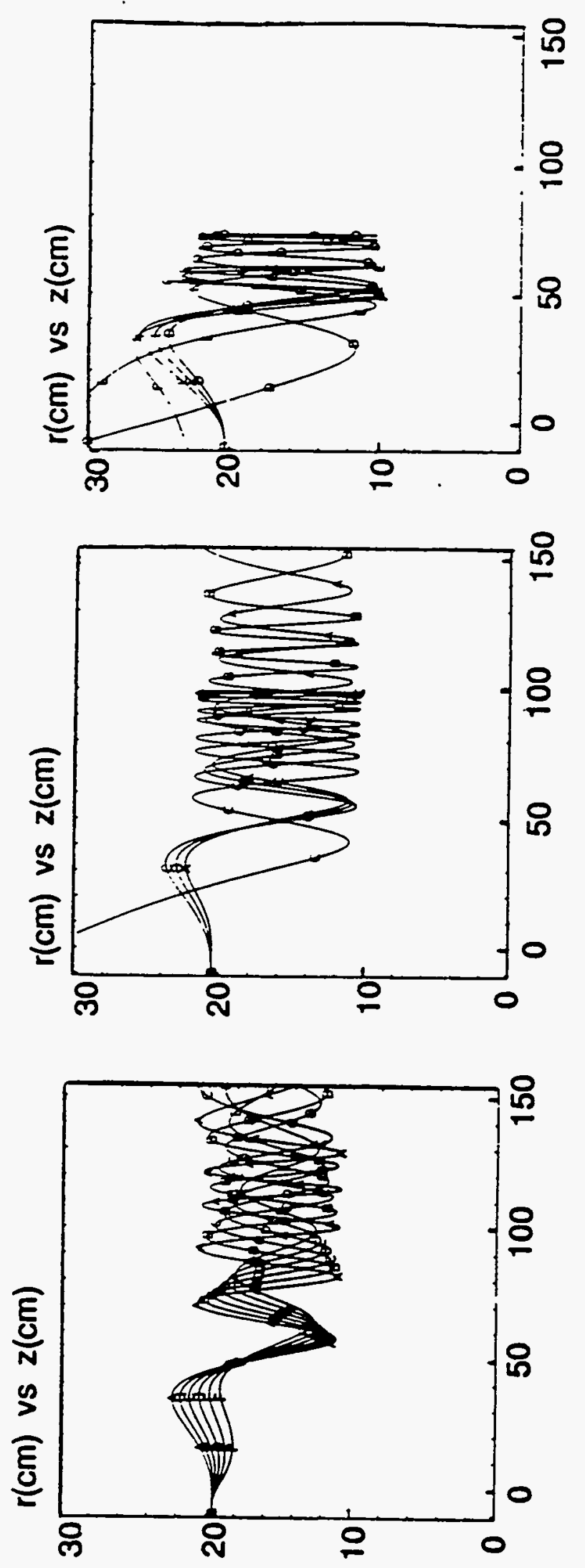

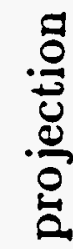

욕 영

. 명 풍

원

岁的约 :

$\rightarrow$ 응 \&

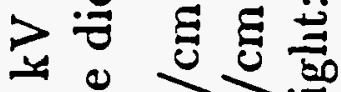

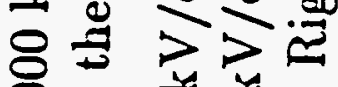

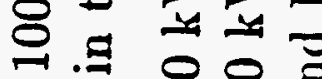

ஸे 의 의

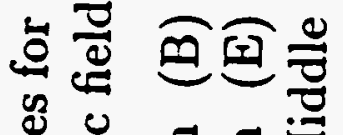

- 붕 영 영

式造 $\vec{x}$

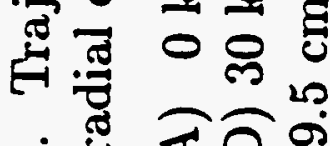

จุ๋

药

象

도 으 


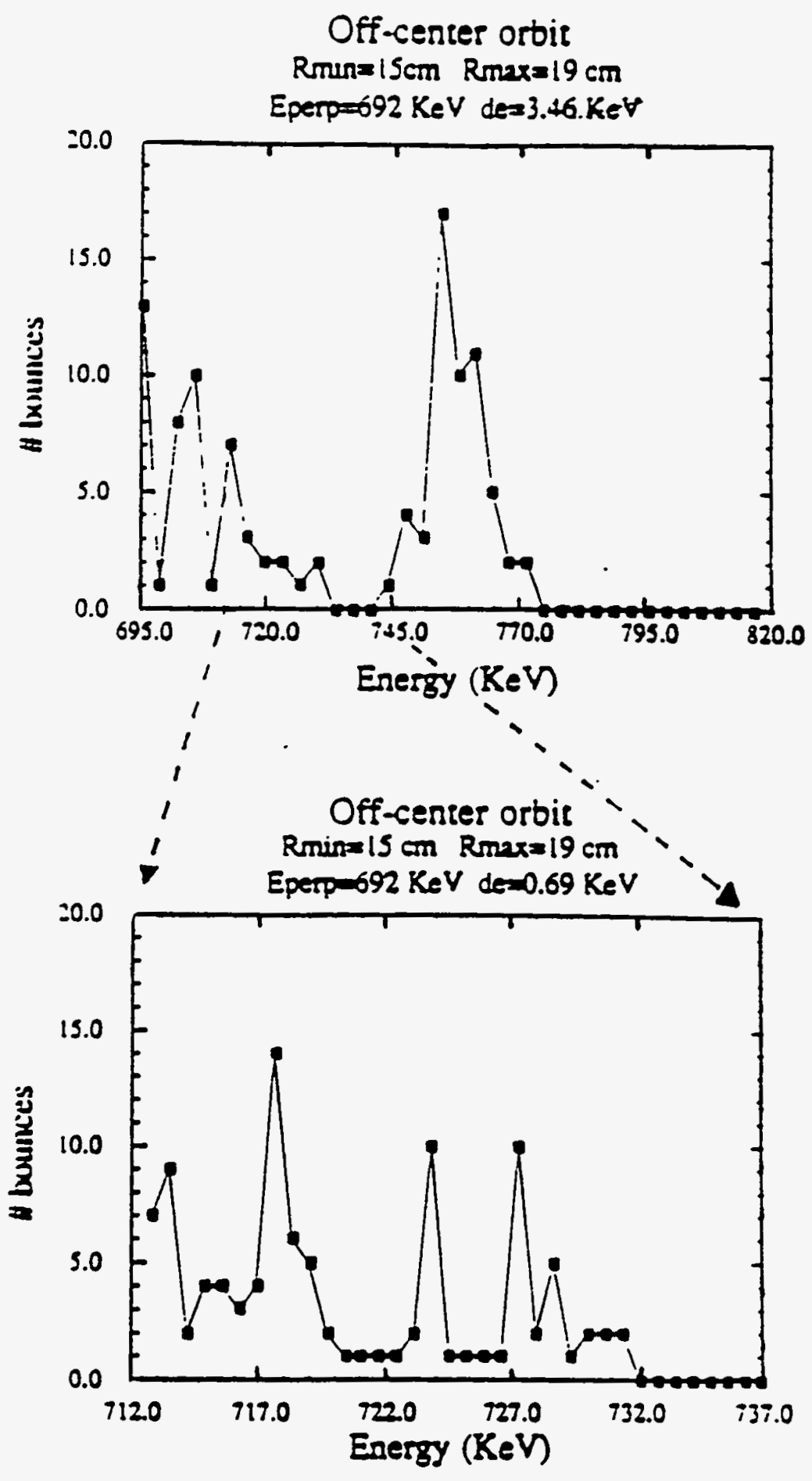

Figure 2-38. Number of axial bounces made by "centered orbit" particles injected with various axial energies.

Top: $\mathcal{E}_{\perp}=612 \mathrm{kV}, r_{0}=16$.

Bottom: $\mathcal{E}_{\perp}=1052 \mathrm{kV}$. 

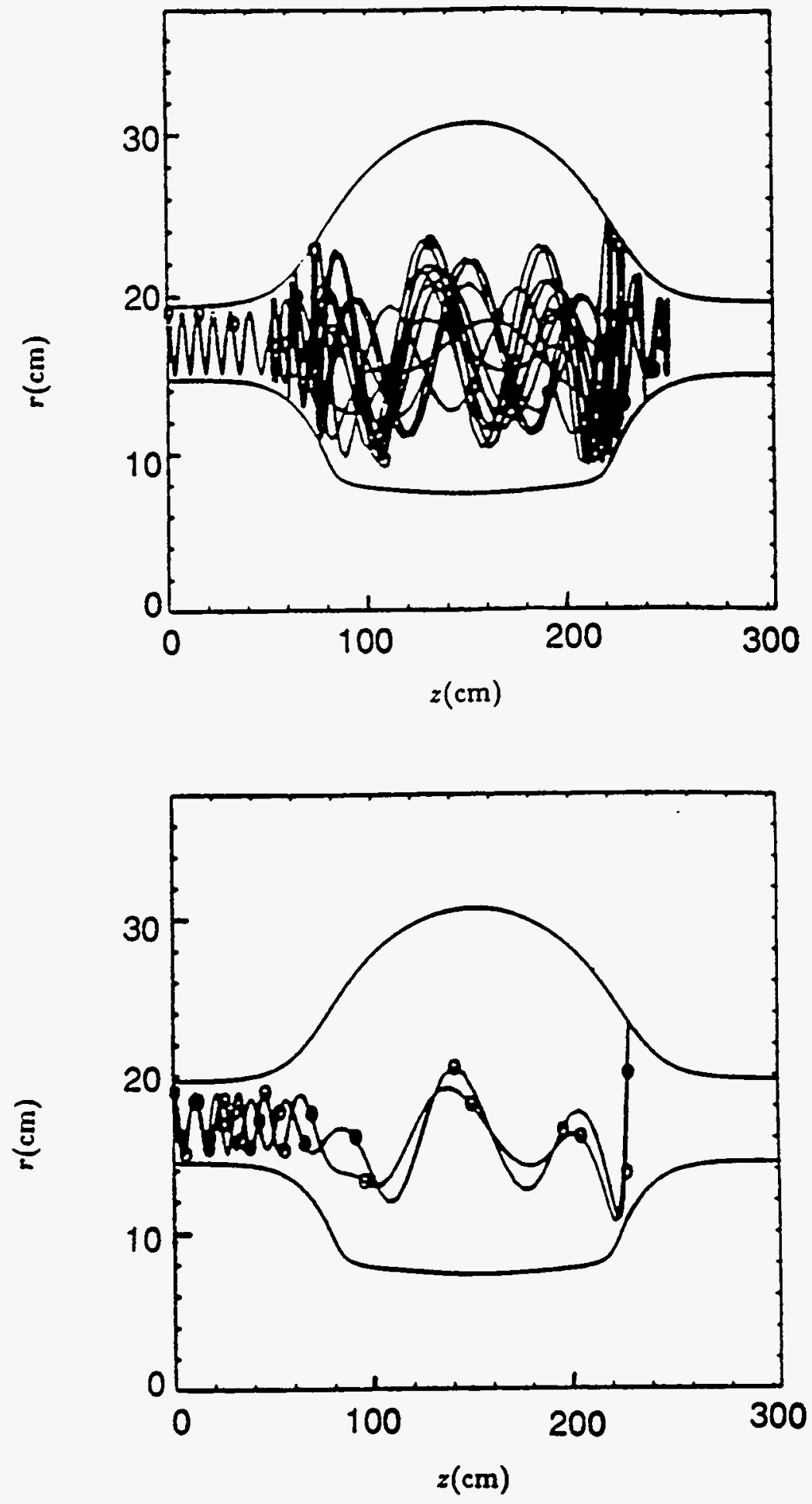

Figure 2-37. Example particle trajectories in an FRC for particles injected with low axial velocity. The particles are injected from $r=19 \mathrm{~cm}$ with $\mathcal{E}_{\perp}=692 \mathrm{kV}$ and parallel energies of $3 \mathrm{kV}$ (top) and $6.5 \mathrm{kV}$ (bottom). The smooth curves bounding the motion are the $\mathcal{H}-\mathcal{P}_{\theta}$ contours for the given particles. 


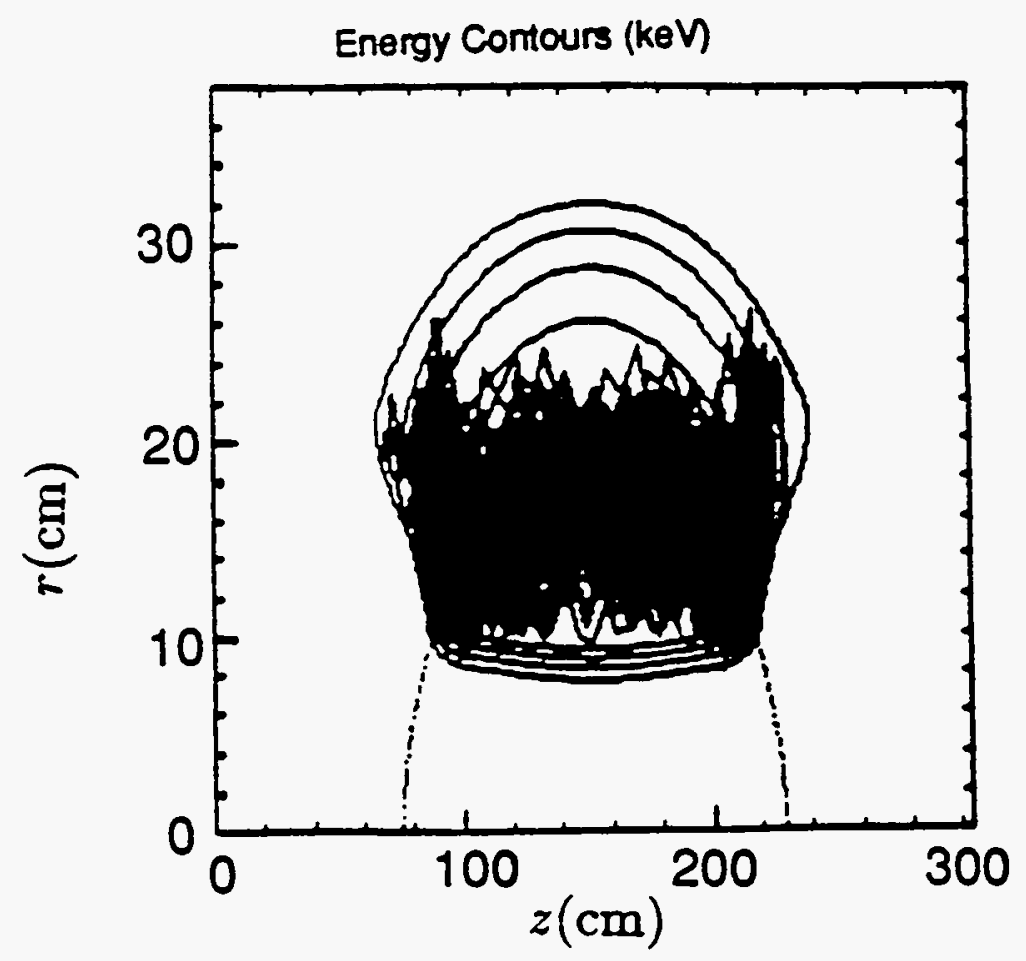

- Figure 2-41.

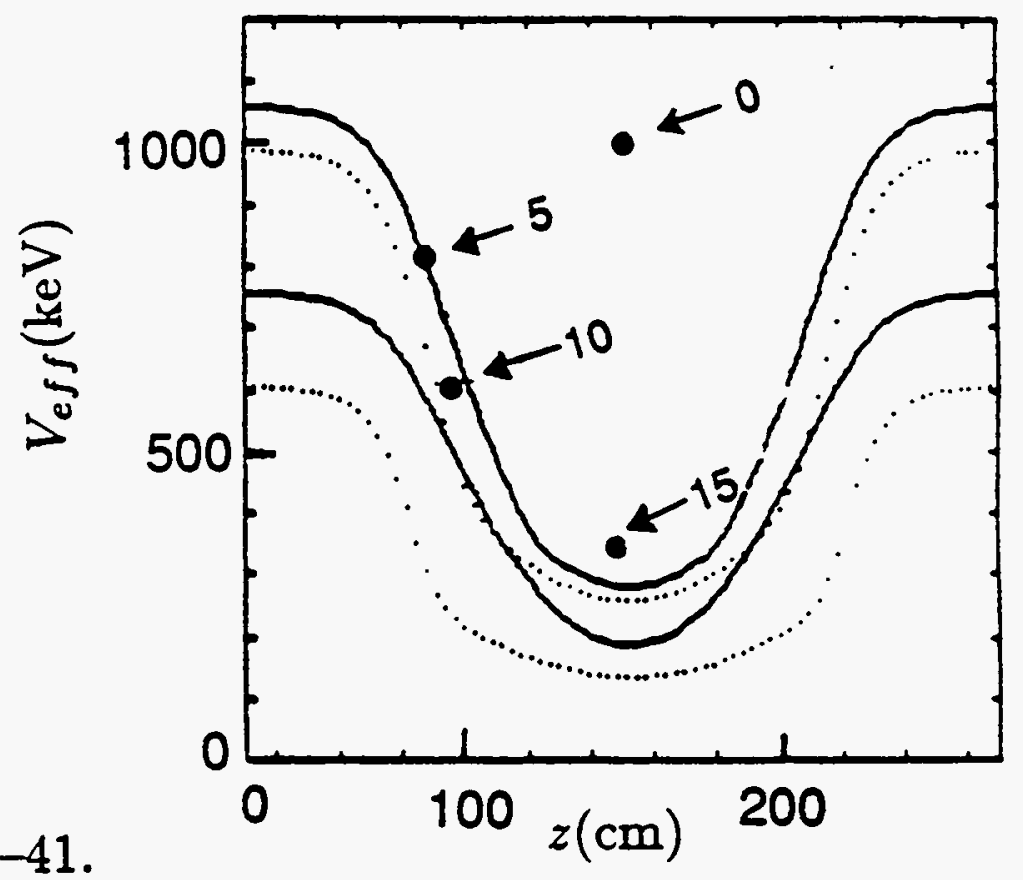

Top: Particle trajectory in the $r-z$ plane for the same conditions as the previous figure. The solid curves are the $\mathcal{H}-\mathcal{P}_{\theta}$ contours for the particle at $0,5,10$, and $15 \mu \mathrm{sec}$. The particle remains trapped.

Bottom: Axial profile of effective potential at the particle's radius for the at selected times. Particle location is indicated by circles. The particle falls further and further below the top of the well. 


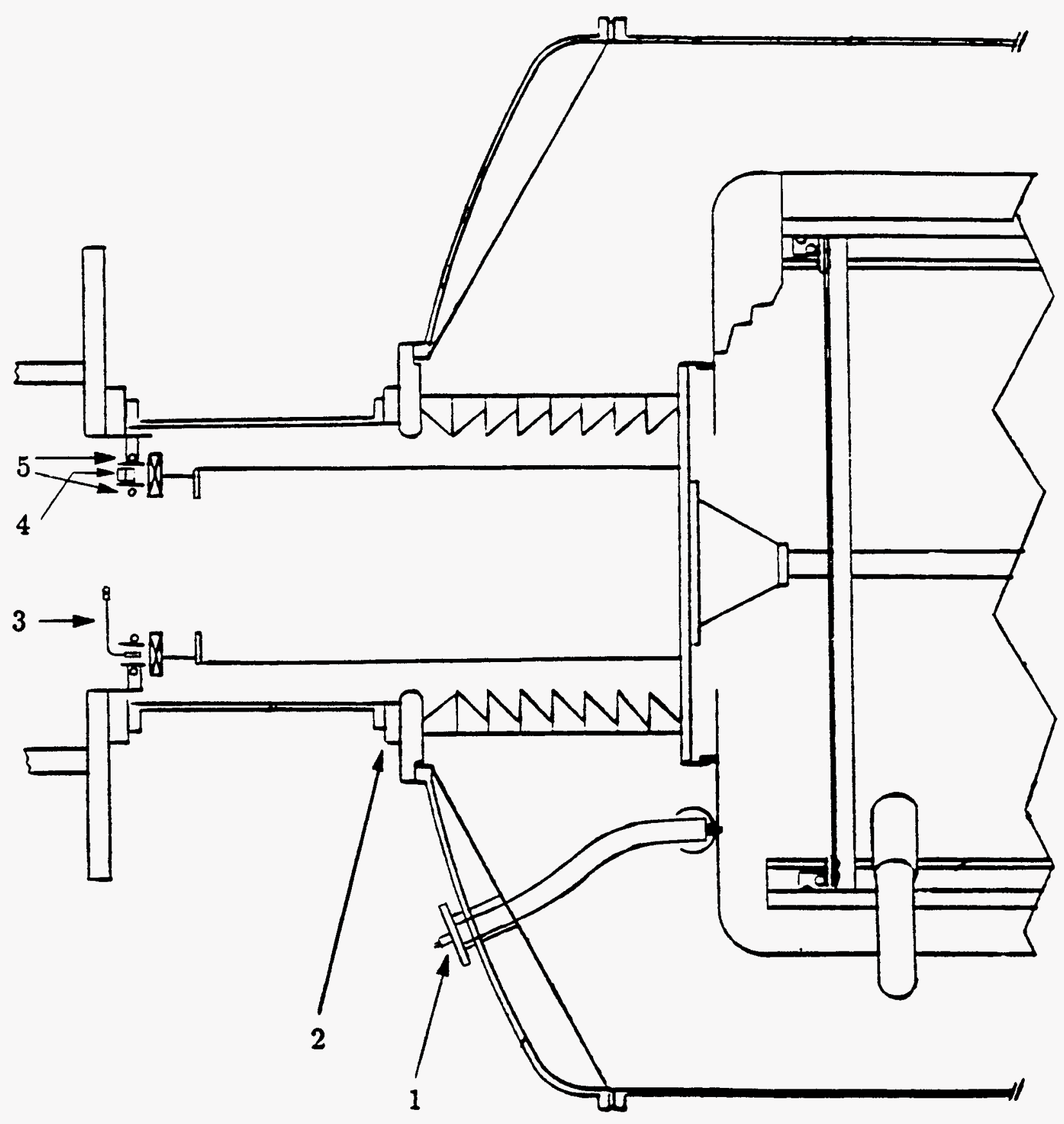

Figure 4-1. The diode area diagnostics.

1. Diode Voltage Monitor 2. Diode Current Monitor

3. Diode Faraday Cups 4. Carbon Activation Target

5. Ion Beam Monitor 


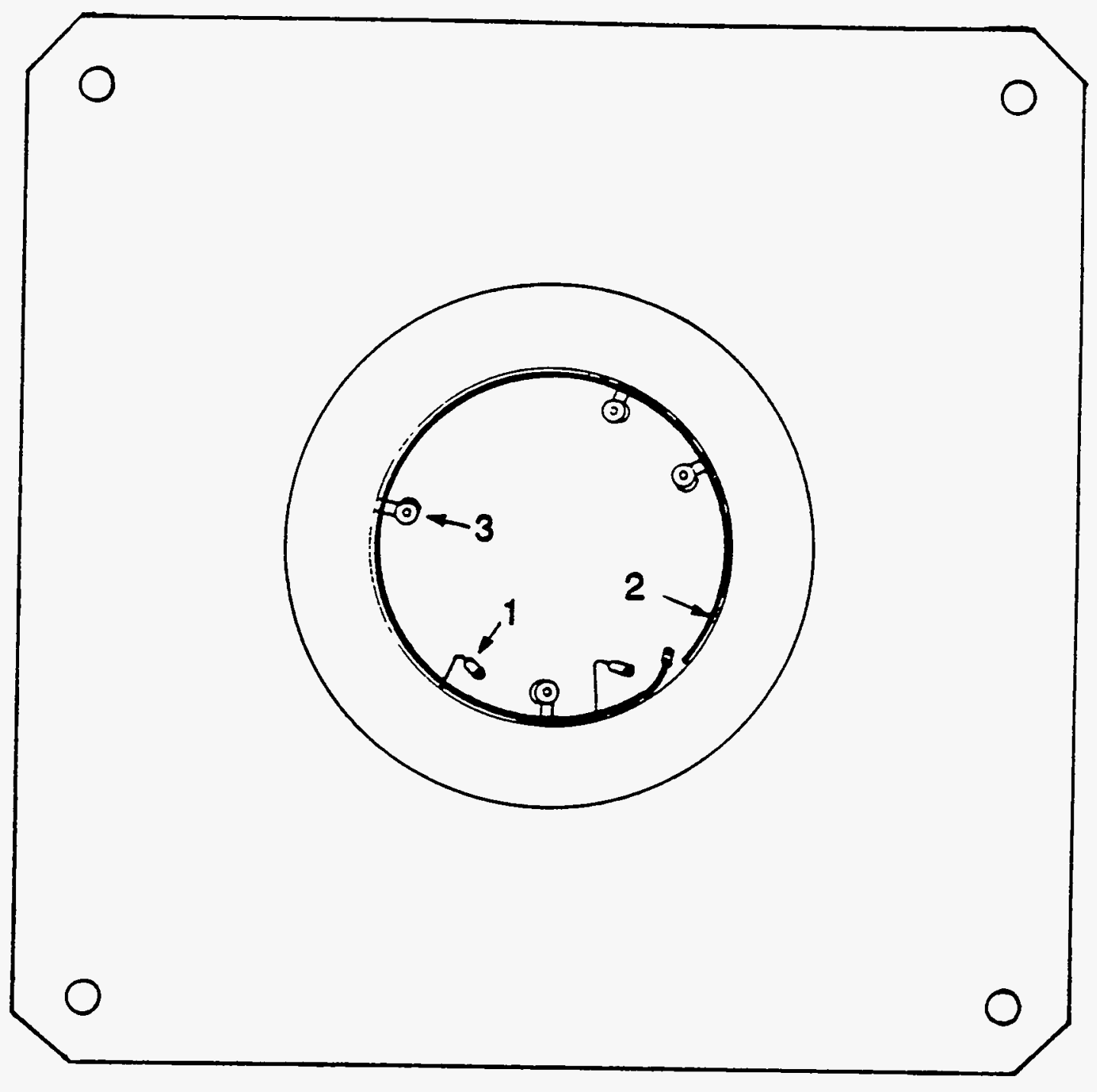

Figure 4-11. The cusp area diagnostics.

1. Cusp Faraday Cups

2. Cusp Rogowski

3. Carbon activation targets 


\section{$-320-$ \\ Vacuum Shots}

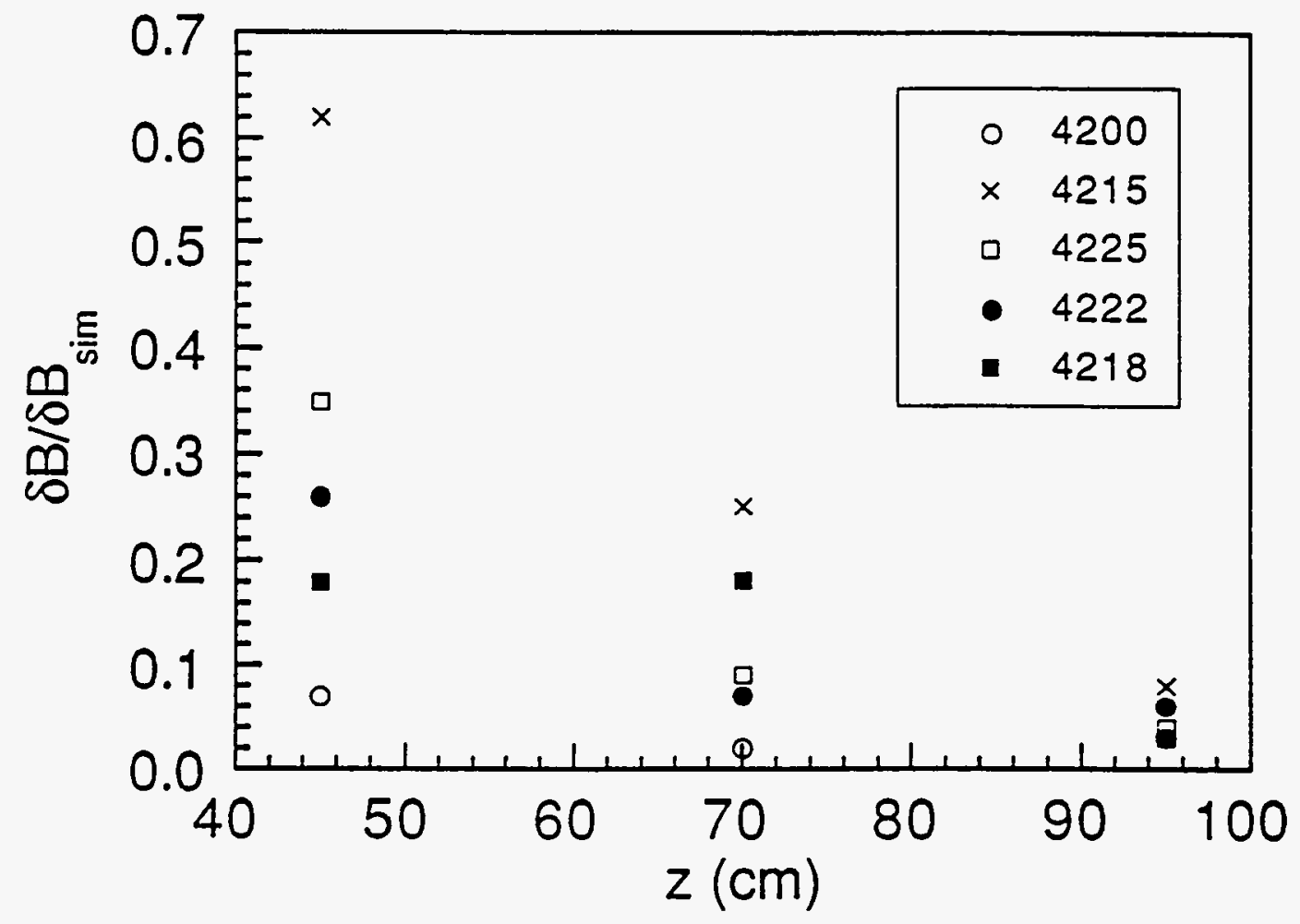

Hydrogen Fill Shots

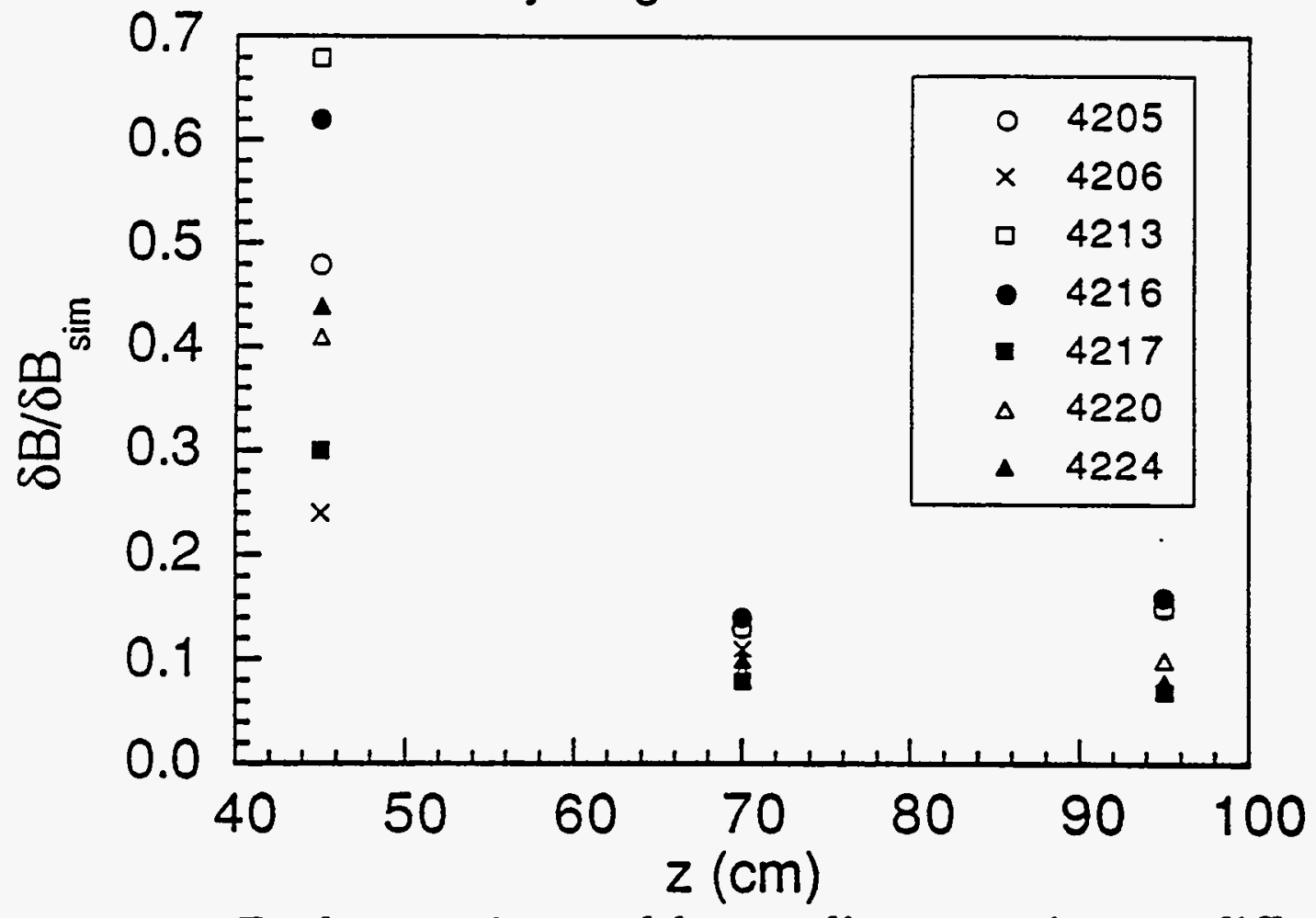

Figure 5-6. Peak experimental beam diamagnetism at different of axial positions, normalized to the peak simulated diamagnetism at the same location.

Top: Vacuum shots.

Bottom: Static fill shots. 